INSTITUT NATIONAL DE RECHERCHE EN INFORMATIQUE ET EN AUTOMATIQUE

\title{
Computing Electronic Structures: a New Multiconfiguration Approach for Excited States
}

Éric CANCÈS — Hervé GALICHER — Mathieu LEWIN

$\mathbf{N}^{\circ} \mathbf{5 2 8 9}$

Aout 2004

Thème NUM 



\title{
Computing Electronic Structures: a New Multiconfiguration Approach for Excited States
}

\author{
Éric CANCĖS*, Hervé GALICHER* ${ }^{*}$, Mathieu LEWIN $^{\dagger}$ \\ Thème NUM - Systèmes numériques \\ Projet MicMac : Méthodes et ingénierie du calcul multi-échelle de l'atome au continuum \\ Rapport de recherche $\mathrm{n}^{\circ} 5289$ - Aout 2004 - 36 pages
}

\begin{abstract}
We present a new method for the computation of electronic excited states of molecular systems. This method is based upon a recent theoretical definition of multiconfiguration excited states (due to one of us, see M. Lewin, Solutions of the Multiconfiguration Equations in Quantum Chemistry, Arch. Rat. Mech. Anal. 171 (2004) 83-114). Contrarily to previously used methods, our algorithm always converges to a stationary state of the multiconfiguration model, which can be interpreted as an approximate excited state of the molecule.

We also compare our method to other approaches and give some explanation of the unsatisfactory behaviours which are sometimes observed when using the latter.

The definition is variational. To compute the first excited state, one has to deform paths on a manifold, like this is usually done in the search for transition states between reactants and products on potential energy surfaces. We propose here a general method for the deformation of paths which could also be useful in other settings.

Numerical results for the special case of two-electrons systems are provided. We compute the first singlet excited state potential energy surface of the $H_{2}$ molecule, and give some numerical results concerning Helium-like atoms.
\end{abstract}

Key-words: Multiconfiguration methods, excited states, time-independent Schrödinger equation, quantum chemistry, moutain pass method, minimax principles, Hartree-Fock theory, Configuration-Interaction method.

* CERMICS and INRIA, École Nationale des Ponts et Chaussées, 6 \& 8 avenue Blaise Pascal, Cité Descartes, 77455 Marne-La-Vallée Cedex 2,France. cances, galicher@cermics.enpc.fr

$\dagger$ CEREMADE, CNRS UMR 7534, Université Paris IX Dauphine, Place du Maréchal de Lattre de Tassigny, 75775 Paris Cedex 16, France.lewin@ceremade.dauphine.fr

\author{
Unité de recherche INRIA Rocquencourt \\ Domaine de Voluceau, Rocquencourt, BP 105, 78153 Le Chesnay Cedex (France) \\ Téléphone : +33139635511 -Télécopie : +33139635330
}




\section{Une nouvelle approche pour le calcul d'états excités électroniques, basée sur les méthodes multi-configurations}

Résumé : Nous présentons une nouvelle méthode pour le calcul d'états excités de molécules dans le cadre des modèles multi-configurations, basée sur une définition récemment introduite par l'un d'entre nous (voir M. Lewin, Solutions of the Multiconfiguration Equations in Quantum Chemistry, Arch. Rat. Mech. Anal. 171 (2004) 83-114). Contrairement aux méthodes existantes, notre algorithme converge toujours vers un état stationnaire du modèle, qui peut être interprété comme un état excité approché de la molécule.

Nous comparons également notre nouveau procédé aux autres approches et tentons d'expliquer les défauts de convergence qui sont parfois observés avec ces dernières.

La définition utilisée est variationnelle. Pour calculer le premier état excité, des chemins doivent être déformés sur une certaine variété, comme pour le calcul d'un état de transition entre réactants et produits sur une surface d'énergie potentielle. Nous proposons ici une méthode générale pour la déformation de chemins, pouvant être utile dans d'autres contextes.

Enfin, nous présentons des résultats numériques pour le cas de systèmes à deux électrons : nous traçons l'énergie du premier état excité de la molécule $H_{2}$ en fonction de la distance inter-atomique, et donnons des résultats pour des atomes à deux électrons comme l'Hélium.

Mots-clés : Méthodes multi-configurations, états excités, équation de Schrödinger, chimie quantique, lemme du col, principes de type min-max, théorie Hartree-Fock, méthodes d'Interactions de Configurations 
Electronic excited states play an essential role in various phenomena of high interest, such as photo-induced chemical reactions, femtosecond spectroscopy, or laser control of molecular processes. Whereas most of the currently used electronic structure models, notably the Hartree-Fock and the Kohn-Sham models, are rigorously founded and quite successful in the description of ground states, their approach to excited states is questionable [20]. The method which seems to be best-adapted to this issue is to date the multiconfiguration selfconsistent field (denoted by MCSCF in the following) method [37, 38, 45]; loosely speaking, this approach leads to variational models which fill the gap between the mean-field HartreeFock and the $N$-body Schrödinger models [38]. However the definition of what actually is an excited state for a nonlinear theory such as MCSCF is still unclear; it is indeed observed that nonlinear electronic structure models have a lot of spurious critical points that cannot be interpreted as approximations of excited states. In other words, solving the equations of the model is clearly not sufficient to obtain a state which really approximates some excited state. In addition, even if we leave aside the above mentioned difficulty, the practical calculation of MCSCF critical points is difficult and numerical algorithms available to date do not always converge. More precisely, they sometimes lead to oscillations between two states of different energies, none of them being a critical point. For all these reasons, the computation of electronic excited states remains one of the main challenges of modern Quantum Chemistry.

In [31], it is emphasized that those difficulties are likely to stem from the currently used definitions of MCSCF excited states that are not correct, for they do not fully take into account the nonlinearity of the model. The purpose of [31] was to provide a more rigorous definition of MCSCF excited states. Our goal in this paper is to show that this theoretical definition can actually be used in practice, at least for the computation of the first excited state.

The paper is organized as follows. In Section 1, we introduce the MCSCF description of electronic structures. In Section 2, we present the new definition of MCSCF excited states and compare it to other definitions currently used in Computational Chemistry. Finally, in Section 3, we describe in details our new algorithm and present numerical results for the case of two-electrons systems.

\section{MCSCF approximation of the time-independent Schrö- dinger equation}

In this section we recall some classical properties of the $N$-body time-independent Schrödinger equation, and briefly present the MCSCF approximation. We refer the reader to $[2,4,31,45]$ for more details.

Let us consider a molecular system consisting of $N$ electrons, and of $M$ nuclei of positive charges $z_{1}, \cdots, z_{M}$. The nuclei are supposed to be correctly described by a classical model and are represented by pointwise charges clamped at positions $\bar{x}_{1}, \cdots, \bar{x}_{M}\left(\bar{x}_{m} \in \mathbb{R}^{3}\right.$ for $1 \leq m \leq M)$. This is the so-called Born-Oppenheimer approximation [3]. The electrons are

$\mathrm{RR} \mathrm{n}^{\circ} 5289$ 
described by the $N$-body quantum Hamiltonian (written in atomic units, see e.g. [4])

$$
H_{N}=\sum_{i=1}^{N}\left(-\frac{1}{2} \Delta_{x_{i}}+V\left(x_{i}\right)\right)+\sum_{1 \leqslant i<j \leqslant N} \frac{1}{\left|x_{i}-x_{j}\right|},
$$

which acts on normalized electronic wavefunctions $\Psi\left(x_{1}, \ldots, x_{N}\right) \in L_{a}^{2}\left(\left(\mathbb{R}^{3}\right)^{N}\right),\|\Psi\|_{L^{2}}=1$. The subscript $a$ indicates that, due to the fermionic nature of the electrons, one solely considers wavefunctions $\Psi$ which are antisymmetric under permutations of variables:

$$
\forall \sigma \in S_{N}, \Psi\left(x_{1}, \ldots, x_{N}\right)=\varepsilon(\sigma) \Psi\left(x_{\sigma(1)}, \ldots, x_{\sigma(N)}\right) \quad \text { a.e. }
$$

Here and below, $S_{N}$ denotes the set of the permutations of the indices $\{1, \ldots, N\}$ and $\varepsilon(\sigma)$ the signature of the permutation $\sigma$. Finally, $V$ is the electrostatic potential generated by the nuclei

$$
V(x)=-\sum_{m=1}^{M} \frac{z_{m}}{\left|x-\bar{x}_{m}\right|} .
$$

In what follows, we denote by $Z=\sum_{m=1}^{M} z_{m}$ the total nuclear charge which is an integer as we work in atomic units.

For the sake of clarity, we do not take the spin into account in the first two sections of the article, but the following arguments can be straightforwardly adapted to the case of spindependent wavefunctions. The spin will be reintroduced in Section 3, in which numerical examples on real molecular systems will be provided.

The operator $H_{N}$ is self-adjoint in $L_{a}^{2}\left(\left(\mathbb{R}^{3}\right)^{N}\right)$, with domain $H_{a}^{2}\left(\left(\mathbb{R}^{3}\right)^{N}\right)$ and form domain $H_{a}^{1}\left(\left(\mathbb{R}^{3}\right)^{N}\right)$. When $Z>N-1$ (an assumption that we will make throughout this article), it is known [49] that its spectrum $\sigma\left(H_{N}\right)$ has the form

$$
\sigma\left(H_{N}\right)=\left\{E_{N}=\lambda_{1} \leqslant \lambda_{2} \leqslant \cdots \leqslant \lambda_{n} \leqslant \cdots\right\} \cup[\Sigma ;+\infty)
$$

where $\left(\lambda_{i}\right)_{i \geqslant 1}$ are eigenvalues strictly below and which converge to $\Sigma$, the bottom of the essential spectrum. The $N$-body ground state energy is the lowest eigenvalue of $H_{N}$ also defined by

$$
E_{N}=\inf \left\{\left\langle\Psi, H_{N} \Psi\right\rangle, \Psi \in H_{a}^{1}\left(\mathbb{R}^{3 N}\right),\|\Psi\|_{L_{a}^{2}\left(\mathbb{R}^{3 N}\right)}=1\right\} .
$$

The eigenfunctions corresponding to the $\lambda_{i}>E_{N}$ are called excited states. Both the ground states and the excited states obviously solve the time-independent Schrödinger equation

$$
H_{N} \Psi=\lambda_{i} \Psi .
$$

Recall that the excited state energies $\lambda_{d}, d \geq 1$, can be obtained by the Rayleigh-Ritz principle

$$
\lambda_{d}=\min _{\operatorname{dim}(W)=d} \max _{\substack{\Psi \in W,\|\Psi\|_{L^{2}}=1}}\langle\Psi, H \Psi\rangle
$$


where the first minimum is taken over all $d$-dimensional subspaces $W$ of the domain of $H_{N}$.

The Schrödinger equation is a model of extremely high accuracy (except for heavy atoms for which core electrons are relativistic). For systems involving a few (say today six or seven) electrons, a direct Galerkin discretization of problem (3) is possible; such a technique is refered to as Full CI in Computational Chemistry. For larger systems, this direct approach is out of reach, due to the excessive dimension of the space $\mathbb{R}^{3 N}$ on which the wavefunctions are defined, and problem (3) must then be approximated. To date, the most commonly used approximations are the Hartree-Fock model (see e.g. [36]) on the one hand, and the Kohn-Sham model (see e.g. [27, 12]) on the other hand. Both of them have been designed for the calculation of ground states and are not really adapted to the calculation of excited states. On the contrary, the MCSCF approximation can be applied to both ground and excited state calculations.

The MCSCF method is based on the following remark:

$$
L_{a}^{2}\left(\left(\mathbb{R}^{3}\right)^{N}\right)=\bigwedge_{n=1}^{N} L^{2}\left(\mathbb{R}^{3}\right),
$$

an equality which can be explicited in the following way. Consider an orthonormal basis $\left(\varphi_{i}\right)_{1 \leq i<+\infty}$ of $L^{2}\left(\mathbb{R}^{3}\right)$. It is well-known that the sequence $\left(\varphi_{i_{1}} \otimes \cdots \otimes \varphi_{i_{N}}\right)_{1 \leq i_{k}<\infty}$ forms an orthonormal basis of $L^{2}\left(\left(\mathbb{R}^{3}\right)^{N}\right)=\bigotimes_{n=1}^{N} L^{2}\left(\mathbb{R}^{3}\right)$, where by definition

$$
\left(\varphi_{i_{1}} \otimes \cdots \otimes \varphi_{i_{N}}\right)\left(x_{1}, \ldots, x_{N}\right)=\varphi_{i_{1}}\left(x_{1}\right) \cdots \varphi_{i_{N}}\left(x_{N}\right) .
$$

An orthonormal basis of the subspace $L_{a}^{2}\left(\left(\mathbb{R}^{3}\right)^{N}\right)$ of $L^{2}\left(\left(\mathbb{R}^{3}\right)^{N}\right)$ can then be obtained by simply considering the antisymmetrized product $\left(\varphi_{i_{1}} \wedge \cdots \wedge \varphi_{i_{N}}\right)_{1 \leq i_{1}<\cdots<i_{N}<+\infty}$ where $\left(\varphi_{i_{1}} \wedge\right.$ $\left.\cdots \wedge \varphi_{i_{N}}\right)$ denotes the so-called Slater determinant of the $\varphi_{i_{k}}$ :

$$
\begin{aligned}
\left(\varphi_{i_{1}} \wedge \cdots \wedge \varphi_{i_{N}}\right)\left(x_{1}, \ldots, x_{N}\right) & =\frac{1}{\sqrt{N !}} \sum_{\sigma \in S_{N}} \varepsilon(\sigma) \varphi_{i_{1}}\left(x_{\sigma(1)}\right) \cdots \varphi_{i_{N}}\left(x_{\sigma(N)}\right) \\
& =\frac{1}{\sqrt{N !}} \operatorname{det}\left(\varphi_{i_{k}}\left(x_{l}\right)\right) .
\end{aligned}
$$

In other words every antisymmetric wavefunction $\Psi$ is an infinite linear combination of such Slater determinants:

$$
\Psi=\sum_{1 \leq i_{1}<\cdots<i_{N}<+\infty} c_{i_{1} \ldots i_{N}} \varphi_{i_{1}} \wedge \cdots \wedge \varphi_{i_{N}}
$$

the sum being convergent in $L_{a}^{2}\left(\left(\mathbb{R}^{3}\right)^{N}\right)$. Remark that $\|\Psi\|_{L^{2}}=1$ is then equivalent to the condition $\sum_{i_{1}<\cdots<i_{N}}\left|c_{i_{1} \ldots i_{N}}\right|^{2}=1$.

An integer $K \geq N$ being fixed, we now consider the subset of $L_{a}^{2}\left(\left(\mathbb{R}^{3}\right)^{N}\right)$ consisting of the wavefuntions $\Psi$ which are finite linear combinations of the $\left(\begin{array}{c}K \\ N\end{array}\right)$ Slater determinants 
constructed from a set of $K$ orthonormal functions $\left(\varphi_{1}, \cdots, \varphi_{K}\right)$ of $L^{2}\left(\mathbb{R}^{3}\right)$, i.e.

$$
\Psi=\sum_{1 \leq i_{1}<\cdots<i_{N} \leq K} c_{i_{1} \ldots i_{N}} \varphi_{i_{1}} \wedge \cdots \wedge \varphi_{i_{N}} .
$$

The MCSCF approach is a variational method for approximating (3) in which both the coefficients $c_{i_{1} \ldots i_{N}}$ and the functions $\left(\varphi_{1}, \cdots, \varphi_{K}\right)$ are variational parameters. Let us mention incidently that the MCSCF method differs from the Configuration-Interaction (CI) method [35], for in the latter, only the coefficients $c_{i_{1} \ldots i_{N}}$ are variational parameters (in a CI calculation, the functions $\left(\varphi_{1}, \cdots, \varphi_{K}\right)$ are issued from a previous Hartree-Fock or Kohn-Sham calculation and are kept fixed).

Following our purpose to describe the MCSCF approach, we therefore introduce the manifold

$$
\mathcal{M}_{N}^{K}=\left\{(c, \Phi) \in \mathbb{R}^{\left(\begin{array}{l}
K \\
N
\end{array}\right)} \times\left(H^{1}\left(\mathbb{R}^{3}\right)\right)^{K}, \sum_{i_{1}<\cdots<i_{N}}\left|c_{i_{1} \ldots i_{N}}\right|^{2}=1, \int_{\mathbb{R}^{3}} \varphi_{i} \varphi_{j}=\delta_{i j}\right\}
$$

where we have denoted by

$$
c=\left(c_{i_{1} \cdots i_{N}}\right) \in \mathbb{R}^{\left(\begin{array}{l}
K \\
N
\end{array}\right)}, \quad \Phi=\left(\varphi_{1}, \ldots, \varphi_{K}\right) \in H^{1}\left(\mathbb{R}^{3}\right)^{K}
$$

(we arrange the $c_{i_{1} \cdots i_{N}}$ in a column vector $c$ using for instance the lexicographical order). Let us note that the functions $\left(\varphi_{1}, \cdots, \varphi_{K}\right)$ are now requested to have a $H^{1}$ regularity, in order to ensure that the MCSCF energy (see formula (9) below) is well defined. Indeed, the MCSCF energy functional that we denote here by $\mathcal{E}_{N}^{K}$, is defined by the formula

$$
\begin{gathered}
\mathcal{E}_{N}^{K}(c, \Phi)=\left\langle\Psi_{(c, \Phi)}, H_{N} \Psi_{(c, \Phi)}\right\rangle \\
\Psi_{(c, \Phi)}=\sum_{1 \leq i_{1}<\cdots<i_{N} \leq K} c_{i_{1} \ldots i_{N}} \varphi_{i_{1}} \wedge \cdots \wedge \varphi_{i_{N}} .
\end{gathered}
$$

The MCSCF ground state energy is then

$$
E_{N}^{K}=\inf _{\mathcal{M}_{N}^{K}} \mathcal{E}_{N}^{K}
$$

If we write $\Psi_{(c, \Phi)}$ as

$$
\Psi_{(c, \Phi)}=\sum_{1 \leq i_{1}, \ldots, i_{N} \leq K} \alpha_{i_{1} \ldots i_{N}} \varphi_{i_{1}} \otimes \cdots \otimes \varphi_{i_{N}}
$$

with

$$
\begin{aligned}
\alpha_{i_{1} \ldots i_{N}} & =0 \text { if } \#\left\{i_{1} \ldots i_{N}\right\}<N \\
& =\frac{\varepsilon(\sigma)}{\sqrt{N !}} c_{i_{\sigma(1)} \ldots i_{\sigma(N)}} \text { otherwise }
\end{aligned}
$$


where $\sigma$ is the permutation of the indices $\{1,2, \cdots, N\}$ such that $i_{\sigma(1)}<\ldots<i_{\sigma(N)}$, the MCSCF energy functional reads [31]

$$
\begin{aligned}
\mathcal{E}_{N}^{K}(c, \Phi)= & N \sum_{1 \leqslant k_{2}, \ldots, k_{N} \leqslant K} \int_{\mathbb{R}^{3}} \frac{1}{2}\left|\sum_{i=1}^{K} \alpha_{i, k_{2} \ldots k_{N}} \nabla \varphi_{i}\right|^{2}+V\left(\sum_{i=1}^{K} \alpha_{i, k_{2} \ldots k_{N}} \varphi_{i}\right)^{2} \\
& +\frac{N(N-1)}{2} \sum_{1 \leqslant k_{3}, \ldots, k_{N} \leqslant K} \iint_{\mathbb{R}^{6}} \frac{\left(\sum_{1 \leqslant i, j \leqslant K} \alpha_{\left.i, j, k_{3} \ldots, k_{N} \varphi_{i}(x) \varphi_{j}(y)\right)^{2}}\right.}{|x-y|} d x d y .
\end{aligned}
$$

Let us point out that, whereas the Schrödinger energy functional $\left\langle\Psi, H_{N} \Psi\right\rangle$ is quadratic, the MCSCF energy functional is not. Consequently, the MCSCF equations, namely the first order stationarity conditions for the critical points of $\mathcal{E}_{N}^{K}$ on the manifold $\mathcal{M}_{N}^{K}$, will be nonlinear.

Remark that when $K=N$, the set $\mathcal{M}_{N}^{N}$ reduces to the set of Slater determinants and one recovers the celebrated Hartree-Fock approximation [36, 33, 34]. The difference between the Hartree-Fock and the exact (non-relativistic) ground state energy [38]

$$
E^{\mathrm{corr}}=E_{N}^{N}-E_{N}
$$

is called the correlation energy, for it originates from correlations between the positions of individual electrons, which are averaged out by the mean-field Hartree-Fock scheme. Estimating the correlation energy is essential for reliably calculating many of the properties of molecules $[2,20]$, in particular in situations where the Hartree-Fock method fails. Since

$$
\lim _{K \rightarrow+\infty} E_{N}^{K}=E_{N}
$$

the MCSCF method is a method of choice for computing the correlation energy.

Mathematically, it is known that a minimizer of (8) exists, and that the associated wavefunction converges to the ground state of $H_{N}$ as $K$ goes to infinity $[29,16,31]$. A minimizer of (8) can be numerically computed by a Newton-like method $[47,48,45,13$, $39]$ in which the orbitals $\varphi_{i}$ and the coefficients $c$ are optimized simultaneously. For the Hartree-Fock model, efficient numerical methods based on combinations of fixed-point and optimization strategies are available [4]. Unfortunately, such algorithms are specifically designed for solving the Hartree-Fock problem and seem to be difficult to adapt to the more general MCSCF setting.

Remark that in (5), all the Slater determinants that can be built with the functions $\varphi_{i}$ are taken into account. Most often, this cannot be done in practice for $\left(\begin{array}{c}K \\ N\end{array}\right)$ is too large a number. It is then necessary to resort to an additional approximation consisting in dividing the electrons into two groups, the inactive electrons that are supposed to be correctly described by a Hartree-Fock type model, and the active electrons that mostly contribute to the correlation energy, and in using the MCSCF methodology for the active

$\mathrm{RR} \mathrm{n}^{\circ} 5289$ 
electrons only. This is the so-called CASSCF (Complete Active Space Self-Consistent Field) approach [43]. All what we shall mention here can be straightforwardly adapted to the CASSCF setting. In particular, the first excited state of a CASSCF model can be computed using a slightly modified version of the numerical algorithm presented in Section 2.3.

\section{On the definition of MCSCF excited states}

Numerical investigations show that the MCSCF energy $\mathcal{E}_{N}^{K}$ possesses a lot of critical points on the manifold $\mathcal{M}_{N}^{K}$ (this is probably due to the nonlinearity of the energy functional). In [31, Theorem 2 (i)] it is shown how an infinity of critical points can be exhibited, following a variational method previously applied for the Hartree-Fock theory in [34]. It seems very difficult to decide in practice whether some critical point can be interpreted as an excited state of the molecular system (in particular, considering the Morse index ${ }^{1}$ is not enough). To achieve this goal, we need to be able to "follow" the critical points as $K$ grows to infinity, and see whether they actually converge to the true excited states of the linear $N$-body Schrödinger model (3).

\subsection{Currently used definition of MCSCF excited states}

Since there are two sets of parameters (the orbitals $\varphi_{i}$ and the coefficients of $c$ ), the multiconfiguration equations take the following general form

$$
\left\{\begin{array}{l}
\gamma_{i}\left(-\frac{\Delta}{2}+V\right) \varphi_{i}+\sum_{1 \leq j, k, l \leq K} b_{i j k l}\left(\left(\varphi_{j} \varphi_{k}\right) * \frac{1}{|x|}\right) \varphi_{l}=\sum_{j=1}^{K} \lambda_{i j} \varphi_{j}, 1 \leq i \leq K \\
H_{\Phi} \cdot c=\beta c
\end{array}\right.
$$

where the $b_{i j k l}$ are real numbers which can be expressed in terms of $c$ (see [16] and [31], where a compact form of the first equations is also given). The first line of (10) is in fact a system of $K$ nonlinear coupled partial differential equations accounting for the stationarity conditions with respect to $\Phi$; the symmetric matrix $\left(\lambda_{i j}\right)$ is the Lagrange multiplier matrix associated with the orthonormality constraints on $\Phi$. The numbers $\gamma_{i}$ are called the occupation numbers and satisfy $0 \leq \gamma_{i} \leq 1$ (see [31] for details); they are all equal to 1 in the Hartree-Fock method. The second equation conveys the stationarity condition with respect to $c$. As both the energy and the constraints are quadratic with respect to $c$, it has the form of an eigenvalue problem. It follows from (7) that the matrix $H_{\Phi}$ is defined by

$$
\left(H_{\Phi}\right)_{I J}=\left\langle\Phi_{I}, H_{N} \Phi_{J}\right\rangle,
$$

where

$$
\Phi_{I}=\varphi_{i_{1}} \wedge \cdots \wedge \varphi_{i_{N}}, \quad \text { when } \quad I=\left\{i_{1}<\cdots<i_{N}\right\} .
$$

\footnotetext{
${ }^{1}$ Recall that the Morse index of a critical point is the number of negative eigenvalues of the Hessian matrix.
} 
In other words, $H_{\Phi}$ is the $\left(\begin{array}{c}K \\ N\end{array}\right) \times\left(\begin{array}{c}K \\ N\end{array}\right)$ matrix of the quadratic form associated with $H_{N}$ when it is restricted to the $\left(\begin{array}{l}K \\ N\end{array}\right)$-dimensional space

$$
V_{\Phi}=\operatorname{Span}\left(\varphi_{i_{1}} \wedge \cdots \wedge \varphi_{i_{N}}, 1 \leq i_{1}<\cdots<i_{N} \leq K\right) .
$$

Let us introduce $\mu_{d}^{K}(\Phi), d=1, \cdots,\left(\begin{array}{c}K \\ N\end{array}\right)$, the eigenvalues of the matrix $H_{\Phi}$. Obviously,

$$
\lambda_{d} \leq \mu_{d}^{K}(\Phi)
$$

for all $K$ and $d$ such that $d \leq\left(\begin{array}{c}K \\ N\end{array}\right)$, and all $\Phi \in H^{1}\left(\mathbb{R}^{3}\right)^{K}$ such that $\int_{\mathbb{R}^{3}} \varphi_{i} \varphi_{j}=\delta_{i j}$. This inequality suggests the currently used definition of excited state energies $[38,45,48,5,47,39]$

$$
\mu_{d}^{K}=\inf _{\substack{\Phi \in H^{1}\left(\mathbb{R}^{3}\right)^{K} \\ \int_{\mathbb{R}^{3}} \Phi \Phi^{T}=I_{K}}} \mu_{d}^{K}(\Phi),
$$

that is to say, quoting [45], "the MCSCF energy results from minimizing the appropriate eigenvalue of the hamiltonian matrix with respect to orbital variations". It can be shown that the expected condition

$$
\lim _{K \rightarrow \infty} \mu_{d}^{K}=\lambda_{d}
$$

is actually fulfilled. We nevertheless believe that this commonly admitted definition of MCSCF excited state energies is the source of various difficulties of both practical and theoretical nature. We now draw up a list of the latter.

i) Practical difficulties in eigenvalue optimization. Solving problem (11) amounts to minimizing the $d^{\text {th }}$ eigenvalue of a matrix depending on a set of parameters $\Phi$. This is known to be a challenging task. Indeed no completely satisfactory numerical method dedicated to solving such problems is available to date, except for very special cases (for instance when the matrix linearly depends on the parameters, see e.g. [32]). In fact, we shall see in the next paragraph that the algorithms which are currently implemented in the Quantum Chemistry simulation packages are not fully adapted to this issue [45, 48, 5, 47].

ii) Problems of degeneracies and non-existence of stationary points. Serious difficulties can occur when optimizing $\mu_{d}^{K}(\Phi)$, due to a possible loss of differentiability of this function in case of degeneracies. As an illustration, let us simply mention a celebrated example due to Rellich and reported on in [42]: consider the family of $2 \times 2$ matrices $(A(x, y))_{(x, y) \in \mathbb{R}^{2}}$ defined by

$$
A(x, y)=\left(\begin{array}{cc}
-\sin x & \sin y \\
\sin y & \sin x
\end{array}\right)
$$

with eigenvalues $\lambda_{1}(x, y)=-\sqrt{\sin ^{2}(x)+\sin ^{2}(y)}$ and $\lambda_{2}(x, y)=\sqrt{\sin ^{2}(x)+\sin ^{2}(y)}$. The second eigenvalue $\lambda_{2}$ degenerates and is not differentiable at its minimum $\left(x_{0}, y_{0}\right)=(0,0)$. Moreover, it is easily seen that there exists no critical point of the form $(0,0, v) \in \mathbb{R}^{2} \times S^{1}$, of

RR $\mathrm{n}^{\circ} 5289$ 
the associated energy $(x, y, v) \in \mathbb{R}^{2} \times S^{1} \mapsto\langle A(x, y) v, v\rangle$. Coming back to our main context, if no stationary MCSCF state exists at the energy level $\mu_{d}^{K}$, it is not clear whether definition (11) is relevant.

iii) The nonlinearity of $\mathcal{E}_{N}^{K}$ is not fully accounted for. An explanation of the difficulties mentioned above can be given in the following way. According to the RayleighRitz principle, we may write

$$
\mu_{d}^{K}=\inf _{\substack{\Phi \in H^{1}\left(\mathbb{R}^{3}\right)^{K} \\ \int \Phi \Phi^{T}=I_{K}}} \inf _{\substack{\left.W \subset \mathbb{R}^{(}\right) \\ \text {dim } W=d}} \sup _{\substack{c \in W \\\|c\|=1}} \mathcal{E}_{N}^{K}(c, \Phi)=\inf _{A \in \mathcal{A}^{d-1}} \sup _{(c, \Phi) \in A} \mathcal{E}_{N}^{K}(c, \Phi),
$$

where

$$
\mathcal{A}^{d-1}=\left\{f\left(S^{d-1}\right) \times\{\Phi\}, f \in C^{0}\left(S^{d-1}, S^{\left({ }_{N}^{K}\right)-1}\right) \text { odd, } \Phi \in H^{1}\left(\mathbb{R}^{3}\right)^{K}, \int \Phi \Phi^{T}=I_{K}\right\} .
$$

Such an inf - sup method cannot give a priori a critical point of $\mathcal{E}_{N}^{K}$, for the set $\mathcal{A}^{d-1}$ appearing in the outer infimum in (13) is not a homotopy family of dimension $d-1$ [18]. A new inf - sup definition of MCSCF excited states which fully takes into account the nonlinearity of the model is proposed below.

But before proceeding further in this direction, we would like to make some comments on the numerical methods used to solve (11). Following [45, 48, 5, 47], the general form of the numerical algorithms currently used to calculate the $(d-1)^{\text {th }}$ excited state can be summarized as follows:

1. Start with some $(c, \Phi)$ obtained for instance from a previous Hartree-Fock or Configuration-Interaction calculation.

2. Compute the matrix $H_{\Phi}$ of the quadratic form associated to $H_{N}$ on the subspace $V_{\Phi}=\operatorname{span}\left\{\varphi_{i_{1}} \wedge \cdots \wedge \varphi_{i_{N}}, i_{1}<\cdots<i_{N}\right\}$.

3. Find $c^{\prime}$ as the $d^{t h}$ eigenvector of this matrix.

4. This $c^{\prime}$ being fixed, minimize the energy with respect to $\Phi$ to obtain a new $\Phi^{\prime}$.

5 . Replace $(c, \Phi)$ by $\left(c^{\prime}, \Phi^{\prime}\right)$ and return to step 2 .

The main difficulty with this method is that the energy is not necessarily decreasing during the computation; it can in fact oscillate, as this can be easily seen when this algorithm is applied to the following toy problem [31]: find the first excited state for the energy functional

$$
\tilde{\mathcal{E}}(c, \Phi)=c^{T}\left(\begin{array}{cc}
-\sin \Phi & 0 \\
0 & \sin \Phi
\end{array}\right) c
$$

with $c \in S^{1}$ and $\left.\Phi \in\right]-\pi, \pi[$ (an oscillation between $\Phi=-\pi / 2$ and $\Phi=\pi / 2$ is obtained). This phenomenon is observed in practice in MCSCF calculations (see, e.g., [5, page 1092]). This is a severe limitation of the above numerical method. 


\subsection{A new definition of MCSCF excited states}

In this section, we present the new definition of MCSCF excited states introduced in [31]. Let be

$$
\mathcal{B}^{d-1}=\left\{f\left(S^{d-1}\right) \mid f \in C^{0}\left(S^{d-1}, \mathcal{M}_{N}^{K}\right), f(x)=(c, \Phi) \Rightarrow f(-x)=(-c, \Phi)\right\} .
$$

The definition of an excited state energy used in [31] is

$$
\lambda_{d}^{K}=\inf _{B \in \mathcal{B}^{d-1}} \sup _{(c, \Phi) \in B} \mathcal{E}_{N}^{K}(c, \Phi)
$$

and the following result has been established

Theorem 1 (Existence of MCSCF excited states [31]). Assume $Z>N-1$ and $1 \leq d \leq\left(\begin{array}{l}K \\ N\end{array}\right)$. Then there exists a critical point $\left(c_{d}, \Phi_{d}\right)$ of the energy $\mathcal{E}_{N}^{K}$ on $\mathcal{M}_{N}^{K}$, with a Morse index lower than or equal to $d-1$, and which satisfies $\mathcal{E}_{N}^{K}\left(c_{d}, \Phi_{d}\right)=\lambda_{d}^{K}$. Moreover, $\lambda_{d}^{K}$ satisfies

$$
\lambda_{d} \leq \lambda_{d}^{K} \leq \mu_{d}^{K}
$$

and therefore

$$
\lim _{K \rightarrow \infty} \lambda_{d}^{K}=\lambda_{d}
$$

Notice that the inequality $\lambda_{d}^{K} \leq \mu_{d}^{K}$ is a simple consequence of the relation $\mathcal{A}^{d-1} \subset \mathcal{B}^{d-1}$.

This result shows that contrarily to what occurs with the definition (11), one always obtains with (15) a critical point which is solution to the MCSCF equations (10), together with a possibly better estimate of the true excited state energy as shown by (16). We have no general criterion to decide whether the strict inequality $\lambda_{d}^{K}<\mu_{d}^{K}$ holds or not. In our simulations (see section 3.3.1 below), we have observed cases where it is true and other cases for which it is not. One can guess that it holds in practice when, due to a problem of degeneracy, no critical point exists at the level $\mu_{d}^{K}\left(\lambda_{d}^{K}\right.$ is always a critical value by Theorem 1). For the Rellich example defined above (12), a simple calculation indeed shows that, with obvious notations, $-1=\lambda_{2}<\mu_{2}=0$.

Remark. In a non-interacting system, i.e. when the interaction term

$$
\sum_{1 \leqslant i<j \leqslant N} \frac{1}{\left|x_{i}-x_{j}\right|}
$$

in the expression (1) of $H_{N}$ is turned off, one can see that for any $d \leq\left(\begin{array}{l}K \\ N\end{array}\right), \lambda_{d}^{K}=\lambda_{d}$ (i.e. the MCSCF and the Schrödinger excited state energies coincide). Moreover, the critical point which is found in Theorem 1 is precisely in this case the $d^{\text {th }}$ eigenfunction of the $N$-body hamiltonian.

Remark. Notice that when $K=N,\left(\begin{array}{l}K \\ N\end{array}\right)=1$, which implies that Theorem 1 does not provide any definition of excited states for the Hartree-Fock method. This strengthens the

$\mathrm{RR} \mathrm{n}^{\circ} 5289$ 
argument that the Hartree-Fock method is not adapted to excited state calculations [20]. Let us point out that the infinitely many solutions to the Hartree-Fock equations constructed by P.-L. Lions in [34] cannot be interpreted as excited states of the molecule. Indeed, the sequence $\left(\nu_{d}\right)_{d \geq 1}$ of the critical values of [34] satisfies $\nu_{d} \rightarrow 0$ as $d \rightarrow \infty$, whereas $\lambda_{d} \rightarrow \Sigma<0$ when $N \geq 2$ (recall that $\Sigma$ is the bottom of the essential spectrum of $H_{N}$ ). Moreover, using the fact that

$$
\left(\cos \theta \varphi_{1}+\sin \theta \varphi_{2}\right) \wedge\left(-\sin \theta \varphi_{1}+\cos \theta \varphi_{2}\right)=\varphi_{1} \wedge \varphi_{2}
$$

for all $\left(\varphi_{1}, \varphi_{2}\right) \in H^{1}\left(\mathbb{R}^{3}\right)^{2}$ with $\int_{\mathbb{R}^{3}} \varphi_{i} \varphi_{j}=\delta_{i j}$ and $\theta \in[0 ; 2 \pi]$, it can be easily seen that $\nu_{1}=\nu_{2}$ for two-electrons systems. Finally, in a non-interacting system, the $\nu_{d}$ do not equal the $\lambda_{d}$, and they satisfy in particular $\nu_{2 d-1} \geq 2 \lambda_{d}$ when $N=2$. The same remarks apply to the sequence constructed in [31, Theorem 2 (i)]. Let us also mention that a definition of Hartree-Fock excited states has been proposed by J.F. Léon in [30]: the first excited state is defined as a minimizer of the Hartree-Fock energy, restricted to the set of Slater determinants which are orthogonal to the Hartree-Fock ground state, and so on for the higher excited states. Note that the so-obtained excited states are not solutions to the Hartree-Fock equations and we therefore do not know whether this definition can be useful in practice.

\subsection{A new method for the computation of the first excited state}

We can deduce from (15) a new computational approach for the calculation of excited states. In this section, we explain the main lines of this algorithm for the case of the first excited state.

Let us emphasize that the definition (15) is valid for all the excited states for which $1 \leq d \leq\left(\begin{array}{c}K \\ N\end{array}\right)$. However, the exploitation of (15) for numerical purposes when $d>2$ is not obvious (one has to deform surfaces of dimension $d-1$ ), and will be the subject of a future work. For the first excited state (i.e. for $d=2$ ), our definition can be easily transformed into a computational method, that we present here.

Let us first clarify the structure of the set $\mathcal{B}^{1}$ defined in (14). Using the parametrization $t \in[0 ; 2] \rightarrow(\cos (\pi t), \sin (\pi t))$ of $S^{1}$, we see that a function satisfying the conditions of (14) can be written $t \in[0 ; 2] \mapsto(c(t), \Phi(t)) \in \mathcal{M}_{N}^{K}$ with $c(1+t)=-c(t)$ and $\Phi(1+t)=\Phi(t)$. Since $\mathcal{E}_{N}^{K}$ is even with regards to $c$ which means

$$
\mathcal{E}_{N}^{K}(-c, \Phi)=\mathcal{E}_{N}^{K}(c, \Phi)
$$

we obtain

$$
\sup _{t \in[0 ; 2]} \mathcal{E}_{N}^{K}(c(t), \Phi(t))=\sup _{t \in[0 ; 1]} \mathcal{E}_{N}^{K}(c(t), \Phi(t)) .
$$

Therefore, we can rewrite (15) as

$$
\lambda_{2}^{K}=\inf _{(c, \Phi) \in \mathcal{M}_{N}^{K}}\left\{\inf _{\gamma \in \Gamma_{(c, \Phi)}} \sup _{t \in[0 ; 1]} \mathcal{E}_{N}^{K}(\gamma(t))\right\}
$$


where

$$
\Gamma_{(c, \Phi)}=\left\{\gamma \in C^{0}\left([0 ; 1], \mathcal{M}_{N}^{K}\right), \gamma(0)=(c, \Phi), \gamma(1)=(-c, \Phi)\right\} .
$$

Notice that the inf - sup problem which is in brackets in (17) is a mountain-pass problem (between $(c, \varphi)$ and $(-c, \varphi)$ ), similar to those encountered in molecular simulation in the search for transition states between reactants and products on potential energy surfaces $[44,40]$. To compute the term in brackets, one thus has to deform paths, as this is usually done in the latter setting.

We propose to simplify the resolution of problem (17) as follows: we simply assume that a global minimizer of the MCSCF energy $(\bar{c}, \bar{\Phi})$ is also a minimizer of the outer minimization in (17). Therefore, we clamp both ends of the trial paths at $(\bar{c}, \bar{\Phi})$ and $(-\bar{c}, \bar{\Phi})$ respectively, and solve the mountain pass problem

$$
\tilde{\lambda}_{2}^{K}=\inf _{\substack{\gamma \in C^{0}\left([0 ; 1], \mathcal{M}_{N}^{K}\right), \gamma(0)=(\bar{c}, \bar{\Phi}), \gamma(1)=(-\bar{c}, \bar{\Phi})}} \sup _{t \in[0 ; 1]} \mathcal{E}_{N}^{K}(\gamma(t)) .
$$

The equality $\lambda_{2}^{K}=\tilde{\lambda}_{2}^{K}$ will be fulfilled provided there exists a path linking $(\bar{c}, \bar{\Phi})$ and an actual minimizer of the outer minimization of (17), along which the energy does not exceed $\lambda_{2}^{K}$. It is indeed likely to be the case, at least for $K$ large enough. Notice that (18) mimics a well-known formula which allows, in the linear case, to obtain the second eigenfunction $\Psi_{2}$ of $H_{N}$, as a mountain pass point between $\Psi_{1}$ and $-\Psi_{1}$, where $H_{N} \Psi_{1}=\lambda_{1} \Psi_{1}$.

Thus, our algorithm to compute the first excited state can be summarized as follows (details will be given in the next section):

1. Use a Newton-like method to compute a ground state $(\bar{c}, \bar{\Phi})$ of the MCSCF energy.

2. Find the path $\gamma$ on the manifold $\mathcal{M}_{N}^{K}$ such that $\gamma(0)=(\bar{c}, \bar{\Phi})$ and $\gamma(1)=(-\bar{c}, \bar{\Phi})$, which minimizes

$$
\max \mathcal{E}_{N}^{K}(\gamma([0 ; 1])) .
$$

3. The first MCSCF excited state is the state which possesses the highest energy on this optimal path.

In practice, solving the mountain-pass problem (Step 2) is rather demanding in terms of CPU time. Therefore, we shall always choose a not too tight convergence criteria to stop the path optimization step. The state of highest energy on the final path is then used as initial guess in a Newton-like procedure to solve (10).

We have found many algorithms in the literature for the optimization of paths (often applied to the simulation of chemical reactions on potential energy surfaces) $[44,22,23$, $25,24,6,15,26,40,41,11,46]$, some of them being quite peculiar in our opinion. The method that we propose below for the deformation of paths, and which seems to give good results on our problem, is of general concern and could therefore also be useful for some other problems.

$\mathrm{RR} \mathrm{n}^{\circ} 5289$ 


\subsection{Solving the mountain pass problem: a method of deformation of paths}

Let us first point out that solving a mountain pass problem is by no means equivalent to finding a saddle point somewhere "between" two minima. The example of the search of the first excited state of the Helium atom (Section 3.3.1) is an illustration of this statement. In this example indeed, the optimal path obtained with our algorithm contains two saddle points of different energies; an algorithm of saddle point localization could converge toward the one of lower energy, and thus underestimate the mountain pass energy.

The best way for properly solving a mountain pass problem is in fact to deform paths. A mathematical study of an algorithm of this type can be found in $[7,8]$. Our method has been inspired by the one described in these references, but it is not identical (see below). In this section, we present it in the following abstract setting: solve the mountain pass problem on the energy surface defined by the functional $\mathcal{E}$ on the Riemann manifold $\mathcal{M}$ between the two points $M_{0}$ and $M_{0}^{\prime}$ of $\mathcal{M}$, or in other words, find a minimizer of

$$
\inf _{\substack{\gamma \in C^{0}([0 ; 1], \mathcal{M}) \\ \gamma(0)=M_{0}, \gamma(1)=M_{0}^{\prime}}} \max _{t \in[0 ; 1]} \mathcal{E}(\gamma(t)) .
$$

Like in $[22,25,24,11,46]$, the main idea is to sample a given path linking $M_{0}$ and $M_{0}^{\prime}$ with a sequence of points $M_{0}, M_{1}, \cdots, M_{N+1}$ of $\mathcal{M}$, such that $M_{N+1}=M_{0}^{\prime}$. During the optimization process, the number $N$ of points used to represent the current path is not necessarily fixed. In our method, we associate with each sequence $\left(t_{k}, M_{k}\right)_{0 \leq k \leq N+1}$ where $0=t_{0}<t_{1}<\cdots<t_{N}<t_{N+1}=1$ are real numbers and where $M_{0}, M_{1}, \cdots, M_{N+1}$ are points on $\mathcal{M}$, a uniquely defined continuous path $\gamma:[0 ; 1] \rightarrow \mathcal{M}$ which satisfies $\gamma\left(t_{k}\right)=M_{k}$. This is done by selecting once and for all, a convenient interpolation scheme. A possible choice is to take for $\gamma(t)$ some piecewise geodesic curve on the manifold $\mathcal{M}$. Simplest interpolation schemes can also be chosen, for in practice, $M_{k}$ and $M_{k+1}$ will be close together. In some cases, spline-type interpolation functions can also be used.

A sequence $\left(t_{k}, M_{k}\right)_{0 \leq k \leq N+1}$ being given, one can use the gradient field of the functional $\mathcal{E}$ to deform the associated continuous path. A naive approach consists in simply moving each $M_{k}$ in the direction opposite to the gradient with a step-length $\alpha_{k}$. Remark that since the new point $M_{k}^{\prime}$ has to lay on the (curved) manifold $\mathcal{M}$, one has to make precise the statement "in the direction opposite to the gradient". The most intrinsic rule is to move $M_{k}$ on the geodesic curve which spurts out from $M_{k}$ in the direction opposite to the gradient [14]. A simpler alternative is first to move $M_{k}$ in the tangent space, then to project the so-obtained point on the manifold (we shall use this method in our problem).

When this naive procedure is iterated, each point $M_{k}$ falls down in one of the valleys of the function. In $[22,25,24]$, it is suggested to circumvent this problem by linking the points $\left(M_{k}\right)_{0 \leq k \leq N+1}$ with strings or elastic bands. Our main contribution is to propose a simpler but apparently more efficient solution: it consists in first computing the path $\gamma^{\prime}$

INRIA 
associated with $\left(t_{k}, M_{k}^{\prime}\right)$, and then finding new points $\left(t_{k}^{\prime}, M_{k}^{\prime \prime}\right)$ which are better distributed in some sense on the (uniquely defined) continuous path $\gamma^{\prime}$. We have observed that for stability reasons, the points need to be redistributed after each minimization step. We use in addition the following rule: the larger the difference between the maximum of the energy on $\gamma$ and the $\mathcal{E}\left(M_{k}\right)$, the smallest the step-length $\alpha_{k}$ in the direction opposite to the gradient. This simple trick helps in preventing the points $M_{k}$ from falling down in the valleys.

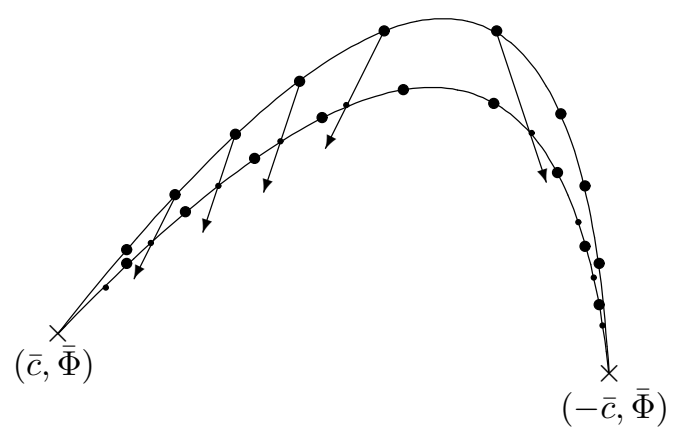

Figure 1: The deformation method

We have applied the above method to several test cases (notably to the ones described in [41]) and we have observed a convergence to the saddle point in all the cases, when the number $N$ of points is large enough. We have also checked on these test cases that switching to a Newton-like method once the mountain-pass algorithm has found a state close enough to the saddle point, is an efficient strategy.

In the following section, we apply this method to the calculation of the first MCSCF excited state.

\section{Computation of the first excited state of two-electrons systems}

\subsection{Singlet and triplet states}

In order to be able to simulate real molecular systems, we now need to reintroduce the spin variables. As the $N$-body hamiltonian $H_{N}$ and the spin operators $S^{2}$ and $S_{z}$ (see e.g. [28]) commute, it is convenient to search for eigenfunctions of $H_{N}$ that also are eigenfunctions of $S^{2}$ and $S_{z}$.

$\mathrm{RR} \quad \mathrm{n}^{\circ} 5289$ 
For two-electron systems, the situation is particularly simple. There are only two types of wavefunctions which are eigenfunctions of both $S^{2}$ and $S_{z}$, namely the so-called singlet and triplet states.

A singlet state is a wavefunction of the form

$$
\Psi^{s}\left(x, \sigma ; y, \sigma^{\prime}\right)=\psi(x, y)|\alpha \beta\rangle\left(\sigma, \sigma^{\prime}\right)
$$

where $\psi(x, y)$ is symmetric in $L^{2}\left(\mathbb{R}^{3} \times \mathbb{R}^{3}\right)$, i.e. such that $\psi(y, x)=\psi(x, y)$. The antisymmetry is carried by the spin function $|\alpha \beta\rangle\left(\sigma, \sigma^{\prime}\right)$ which is defined for $\left(\sigma, \sigma^{\prime}\right) \in\{|\uparrow\rangle,|\downarrow\rangle\} \times$ $\{|\uparrow\rangle,|\downarrow\rangle\}$ by

$$
|\alpha \beta\rangle\left(\sigma, \sigma^{\prime}\right)=\frac{1}{\sqrt{2}}\left(\alpha(\sigma) \beta\left(\sigma^{\prime}\right)-\beta(\sigma) \alpha\left(\sigma^{\prime}\right)\right)
$$

where

$$
\alpha(|\uparrow\rangle)=1, \quad \alpha(|\downarrow\rangle)=0, \quad \beta(|\uparrow\rangle)=0, \quad \beta(|\downarrow\rangle)=1 .
$$

A triplet state takes the form

$$
\Psi^{t}\left(x, \sigma ; y, \sigma^{\prime}\right)=\psi(x, y) \alpha(\sigma) \alpha\left(\sigma^{\prime}\right)
$$

where $\psi(x, y)$ is antisymmetric in $L^{2}\left(\mathbb{R}^{3} \times \mathbb{R}^{3}\right)$, i.e. $\psi(y, x)=-\psi(x, y)$ (the spin function $\alpha(\sigma) \alpha\left(\sigma^{\prime}\right)$ is symmetric and the antisymmetry is carried by the function of the space variables).

For two electron systems, the MCSCF wavefunctions thus read

$$
\psi=\sum_{1 \leq i, j \leq K} c_{i j} \varphi_{i} \otimes \varphi_{j}
$$

where the $K \times K$ matrix $C=\left(c_{i j}\right)$ is symmetric for singlet states and antisymmetric for triplet states. The condition $\|\psi\|_{L^{2}}=1$ also reads $\|C\|=1$ where $\|C\|=\operatorname{tr}\left(C C^{T}\right)^{1 / 2}$.

For numerical simulations, one most often resorts to a Galerkin approximation. One expands each $\varphi_{i}$ on a finite basis $\left(\chi_{\mu}\right)_{1 \leq \mu \leq N_{b}}$ of $H^{1}\left(\mathbb{R}^{3}\right)$ functions specially designed for electronic structure calculations, the so-called atomic orbitals. This approximation is refered to as the Linear Combination of Atomic Orbitals (LCAO) approximation in the Computational Chemistry literature (see e.g. [21]). Let $S$ be the matrix defined by

$$
S_{\mu \nu}=\int_{\mathbb{R}^{3}} \chi_{\mu} \chi_{\nu}
$$

and $\varphi=\left(\varphi_{\mu i}\right)$ be the $N_{b} \times K$ coordinate matrix of the functions $\left(\varphi_{i}\right)_{1 \leq i \leq K}$ in the basis $\left(\chi_{\mu}\right)_{1 \leq \mu \leq N_{b}}$. The condition $\int_{\mathbb{R}^{3}} \varphi_{i} \varphi_{j}=\delta_{i j}$ also reads $\varphi^{T} S \varphi=I_{K}$ (of course, $N_{b}$ must be chosen greater or equal to $K$ ) and the energy of a state $\Psi^{s}$ or $\Psi^{t}$, as a function of $C$ and $\varphi$, has the following expression:

$$
\mathcal{E}(C, \varphi)=2 \operatorname{tr}\left(C^{T} \varphi^{T} h \varphi C\right)+\operatorname{tr}\left(\left(\varphi C \varphi^{T}\right)^{T} \mathcal{W}\left(\varphi C \varphi^{T}\right)\right),
$$


where $h$ is the $N_{b} \times N_{b}$ matrix defined by

$$
h_{\mu \nu}=\frac{1}{2} \int_{\mathbb{R}^{3}} \nabla \chi_{\mu} \cdot \nabla \chi_{\nu}+\int_{\mathbb{R}^{3}} V \chi_{\mu} \chi_{\nu}
$$

and where $\mathcal{W}$ is the linear map associated with the tensor $W$ defined by

$$
W_{\mu \nu \kappa \lambda}=\int_{\mathbb{R}^{3} \times \mathbb{R}^{3}} \frac{\chi_{\mu}(x) \chi_{\nu}(y) \chi_{\kappa}(x) \chi_{\lambda}(y)}{|x-y|} d x d y
$$

i.e. for any $N_{b} \times N_{b}$ matrix $X$

$$
[\mathcal{W}(X)]_{\mu \nu}=\sum_{\kappa, \lambda=1}^{N_{b}} W_{\mu \nu \kappa \lambda} X_{\kappa \lambda} .
$$

Remark that expression (20) is valid for both singlet and triplet states, but that the matrix $C$ appearing in this formula is symmetric for singlet states and antisymmetric for triplet states.

For the sake of brevity, we only deal with the singlet state case. The manifold of admissible singlet states is

$$
\mathcal{M}=\left\{(C, \varphi) \in M(K, K) \times M\left(N_{b}, K\right), C^{T}=C, \operatorname{tr}\left(C^{T} C\right)=1, \varphi^{T} S \varphi=I_{K}\right\},
$$

where $M\left(K, K^{\prime}\right)$ denotes the set of $K \times K^{\prime}$ matrices. The MCSCF equations (i.e. the stationarity conditions of the MCSCF energy (20) on the manifold $\mathcal{M}$ ) take the form

$$
\left\{\begin{array}{l}
\left(\varphi^{T} h \varphi C+C^{T} \varphi^{T} h \varphi\right)+\varphi^{T} \mathcal{W}\left(\varphi C \varphi^{T}\right) \varphi=\beta C \\
h \varphi C C^{T}+\mathcal{W}\left(\varphi C \varphi^{T}\right) \varphi C=S \varphi \Lambda
\end{array}\right.
$$

where $\beta \in \mathbb{R}$ and where $\Lambda$ is a $K \times K$ symmetric matrix.

The problem can be dramatically simplified by using a rotation invariance property. Indeed, using the constraint $\varphi^{T} S \varphi=I_{K}$, we see that $\mathcal{E}(C, \varphi)$ only depends on $X=\varphi C \varphi^{T}$ :

$$
\mathcal{E}(C, \varphi)=2 \operatorname{tr}\left(S X^{T} h X\right)+\operatorname{tr}\left(X^{T} \mathcal{W}(X)\right) .
$$

Notice now that for any rotation matrix $U \in O_{K}(\mathbb{R})$, one has $X=\varphi C \varphi^{T}=\varphi^{\prime} C^{\prime} \varphi^{\prime T}$ with $\varphi^{\prime}=\varphi U$ and $C^{\prime}=U^{T} C U$. Since $\varphi^{\prime}$ obviously satisfies the constraint $\varphi^{\prime T} S \varphi^{\prime}=I_{K}$, we see that the energy functional $\mathcal{E}$ is invariant under the action $U \cdot(C, \varphi)=\left(U^{T} C U, \varphi U\right)$ of the orthogonal group $O_{K}(\mathbb{R})$. Since $C$ is symmetric and real, there exists a $U \in O_{K}(\mathbb{R})$ such that $C^{\prime}=\operatorname{diag}\left(c_{1}, \ldots, c_{K}\right)$. Up to a rotation of the orbitals, $\varphi^{\prime}=\varphi U$, this means that there is no restriction in assuming that the matrix $C$ is diagonal. When using this reduced model (which is not an approximation), the manifold of admissible singlet states reads

$$
\mathcal{M}^{\text {red }}=S^{K-1} \times \mathcal{W}_{K}^{N_{b}}
$$

RR $n^{\circ} 5289$ 
with

$$
\mathcal{W}_{K}^{N_{b}}=\left\{\varphi \in M\left(N_{b}, K\right), \varphi^{T} S \varphi=I_{K}\right\},
$$

and the energy functional is given by

$$
\mathcal{E}^{\mathrm{red}}(c, \varphi)=\mathcal{E}\left(C^{s}(c), \varphi\right)
$$

where $C^{s}(c)=\operatorname{diag}\left(c_{1}, \ldots, c_{K}\right)$. Lastly, the MCSCF equations become

$$
\left\{\begin{array}{l}
H(\varphi) \cdot c=\beta \cdot c \\
h \varphi\left(C^{s}(c)\right)^{2}+\mathcal{W}\left(\varphi C^{s}(c) \varphi^{T}\right) \varphi C^{s}(c)=S \varphi \Lambda
\end{array}\right.
$$

with

$$
H(\varphi)_{i j}=2 \varphi_{i}^{T} h \varphi_{i} \delta_{i j}+\varphi_{i}^{T} \mathcal{W}\left(\varphi_{j} \varphi_{j}^{T}\right) \varphi_{i} .
$$

Notice that the same reduction can be done in $\mathcal{M}_{2}^{K}$ (see [16, 1, 9, 10] and [31, Appendix]). Triplet states can also be simplified with the same type of argument since for any antisymmetric matrix $C$, there exists a rotation matrix $U$ such that $U^{T} C U=C^{\prime}=\operatorname{diag}\left(C_{1}, \ldots, C_{p}\right)$ if $K=2 p$, and $C^{\prime}=\operatorname{diag}\left(C_{1}, \ldots, C_{p}, 0\right)$ if $K=2 p+1$, where

$$
C_{i}=\frac{1}{\sqrt{2}}\left(\begin{array}{cc}
0 & c_{i} \\
-c_{i} & 0
\end{array}\right)
$$

\subsection{Description of the algorithm}

For the sake of brevity, we only deal with the reduced model of the singlet state.

We shall make use of the following interpolation rule: a discrete path on $\mathcal{M}^{\text {red }}=S^{K-1} \times$ $\mathcal{W}_{K}^{N_{b}}$ being given as a finite sequence $\left(t_{k},\left(c^{k}, \varphi^{k}\right)\right)_{0 \leq k \leq N+1}$ where

- $t_{0}=0<t_{1}<\cdots<t_{N}<t_{N+1}=1$ are real numbers ;

- $\left(c^{k}, \varphi^{k}\right)_{0 \leq k \leq N+1}$ are points of $S^{K-1} \times \mathcal{W}_{K}^{N_{b}}$,

we define the associated continuous path $\gamma \in C^{0}\left([0,1], S^{K-1} \times \mathcal{W}_{K}^{N_{b}}\right)$ according to

$$
\forall t \in[0,1], \quad \gamma(t)=(c(t), \varphi(t))
$$

where

$$
\forall 0 \leq k \leq N, \quad \forall t \in\left[t_{k}, t_{k+1}\right], \quad c(t)=\cos \left(\theta_{k}(t)\right) c^{k}+\sin \left(\theta_{k}(t)\right) \widetilde{c}^{k+1}
$$

with

$$
\left\{\begin{array}{l}
\widetilde{c}^{k+1}=\frac{c^{k+1}-\left(c^{k+1}, c^{k}\right) c^{k}}{\left\|c^{k+1}-\left(c^{k+1}, c^{k}\right) c^{k}\right\|} \\
\theta_{k}(t)=\frac{t-t_{k}}{t_{k+1}-t_{k}} \arccos \left(c^{k+1}, c^{k}\right) .
\end{array}\right.
$$

INRIA 
and

$$
\forall 0 \leq k \leq N, \quad \forall t \in\left[t_{k}, t_{k+1}\right], \quad \varphi(t)=\widetilde{\varphi}(t)\left[\widetilde{\varphi}(t)^{T} S \widetilde{\varphi}(t)\right]^{-1 / 2}
$$

with

$$
\widetilde{\varphi}(t)=\varphi^{k}+\frac{t-t_{k}}{t_{k+1}-t_{k}}\left(\varphi^{k+1}-\varphi^{k}\right) .
$$

We can now describe our algorithm for computing the first excited state of two-electron systems.

Step A: search for a MCSCF ground state $(\bar{c}, \bar{\varphi})$, i.e. solve

$$
\inf \left\{\mathcal{E}^{\mathrm{red}}(c, \varphi), \quad(c, \varphi) \in S^{K-1} \times \mathcal{W}_{K}^{N_{b}}\right\} .
$$

with the Newton-like algorithm described in Appendix; a convenient initial guess is the Hartree-Fock ground state, which can itself be obtained by a self-consistent field algorithm [4].

\section{Step B: construction of an initial trial path.}

As already mentioned in Section 2.3, we get rid of the outer minimization in (17) and concentrate on solving

$$
\lambda_{2}^{s, r}=\inf _{\substack{\gamma \in C^{0}\left([0,1], \mathcal{M}^{\mathrm{red}}\right) \\ \gamma(0)=(\bar{c}, \bar{\varphi}), \gamma(1)=(-\bar{c}, \bar{\varphi})}} \max _{t \in[0 ; 1]} \mathcal{E}^{\mathrm{red}}(\gamma(t)) .
$$

Let $\bar{c}_{1}$ be the second eigenvector of the hamiltonian matrix

$$
[H(\bar{\varphi})]_{i j}=2 \bar{\varphi}_{i}^{T} h \bar{\varphi}_{i} \delta_{i j}+\bar{\varphi}_{i}^{T} \mathcal{W}\left(\bar{\varphi}_{j} \bar{\varphi}_{j}^{T}\right) \bar{\varphi}_{i}
$$

(note that $\bar{c}$ is the ground state of $H(\bar{\varphi})$ ). A possible initial trial path is a path on which the parameter $\varphi$ is constant, for instance

$$
\gamma^{0}(t)=(c(t), \bar{\varphi})
$$

with $c(t)=\cos (t \pi) \bar{c}+\sin (t \pi) \bar{c}_{1}$. A better initial guess can however be obtained by random perturbations of that reference path. In practice, we randomly choose a collection of $N_{\text {sto }}$ states $\left(\bar{c}_{j}^{\prime}, \bar{\varphi}_{j}^{\prime}\right) \in\left(\operatorname{vect}(\bar{c})^{\perp} \cap S^{K-1}\right) \times \mathcal{W}_{K}^{N_{b}}$ such that for all $j$

$$
\left\|\bar{c}_{j}^{\prime}-\bar{c}_{1}\right\| \leq \varepsilon\left\|\bar{c}_{1}\right\| \text { and }\left\|\bar{\varphi}_{j}^{\prime}-\bar{\varphi}\right\| \leq \varepsilon\|\bar{\varphi}\|
$$

for a small $\varepsilon$, and we consider the $N_{\text {sto }}$ continuous paths $\gamma^{j}(t)$ associated with the three-point discrete paths

$$
\gamma^{j}(0)=(\bar{c}, \bar{\varphi}), \gamma^{j}(1 / 2)=\left(\bar{c}_{j}^{\prime}, \bar{\varphi}_{j}^{\prime}\right), \gamma^{j}(1)=(-\bar{c}, \bar{\varphi}) .
$$

We then selects, among the $N_{\text {sto }}$ paths $\gamma^{j}$, the path $\gamma_{0}(t)$ for which the maximal energy $\max \mathcal{E}^{\mathrm{red}}\left(\gamma^{j}([0 ; 1])\right)$ is minimum. The above method can obviously be generalized to discrete 
paths containing more than three points and can also be used to improve the following step $\mathrm{C}$ (path optimization) when necessary.

We then set $m=0, t_{k}=k /(N+1), \gamma_{0}^{k}=\left(c^{k, 0}, \varphi^{k, 0}\right)=\gamma_{0}\left(t_{k}\right)$ for $1 \leq k \leq N$, and

$$
\begin{gathered}
E_{0}^{\min }=\mathcal{E}^{\mathrm{red}}(\bar{c}, \bar{\varphi}), \quad E_{0}^{\max }=\max _{1 \leq k \leq N} \mathcal{E}^{\mathrm{red}}\left(\gamma_{0}^{k}\right), \\
i_{0}^{\max }=\underset{1 \leq k \leq N}{\operatorname{argmax}} \mathcal{E}^{\mathrm{red}}\left(\gamma_{0}^{k}\right) .
\end{gathered}
$$

\section{Step C: path optimization.}

For the sake of simplicity, we displace the nodes in the direction opposite to the gradient; for this purpose,

1. we compute for each $1 \leq k \leq N$, the MCSCF energy at the point $\gamma_{m}^{k}=\left(c^{k, m}, \varphi^{k, m}\right)$

$$
E_{m}^{k}=\mathcal{E}^{\mathrm{red}}\left(\gamma_{m}^{k}\right)=\left(H\left(\varphi^{k, m}\right) c^{k, m}, c^{k, m}\right)
$$

2. we project the components $\nabla_{c} \mathcal{E}^{\text {red }}\left(\gamma_{m}^{k}\right)$ and $\nabla_{\varphi}^{S} \mathcal{E}^{\text {red }}\left(\gamma_{m}^{k}\right)$ of the gradients of the energy at the points $\gamma_{m}^{k}$ on the tangent spaces of the underlying manifolds:

$$
\begin{gathered}
g_{m}^{k}=\nabla_{c} \mathcal{E}^{\mathrm{red}}\left(c^{k, m}, \varphi^{k, m}\right)-\left(\nabla_{c} \mathcal{E}^{\mathrm{red}}\left(c^{k, m}, \varphi^{k, m}\right), c^{k, m}\right) c^{k, m} \\
=2\left(H\left(\varphi^{k, m}\right)-E_{m}^{k}\right) c^{k, m} ; \\
G_{m}^{k}=4 P_{\varphi^{k, m}}^{S}\left[S^{-1}\left(h \varphi^{k, m} C^{s}\left(c^{k, m}\right)^{2}+\mathcal{W}\left(\varphi^{k, m} C^{s}\left(c^{k, m}\right)\left(\varphi^{k, m}\right)^{T}\right) \varphi^{k, m} C^{s}\left(c^{k, m}\right)\right)\right],
\end{gathered}
$$

where $P_{\varphi^{k, m}}^{S}$ is the orthogonal projector of $M\left(N_{b}, K\right)$ on the tangent space $T_{\varphi^{k, m}} \mathcal{W}_{K}^{N_{b}}$ for the scalar product $\langle\cdot, \cdot\rangle_{S}$ (defined on $M\left(N_{b}, K\right)$ by $\langle A, B\rangle_{S}=\operatorname{tr}\left(A^{T} S B\right)$ ). One has

$$
\forall \varphi \in \mathcal{W}_{K}^{N_{b}}, \quad \forall Z \in M\left(N_{b}, K\right), \quad P_{\varphi}^{S} Z=Z-\frac{1}{2} \varphi\left(\varphi^{T} S Z+Z^{T} S \varphi\right)
$$

3. we set

$$
E_{m}^{\max }=\max _{1 \leq k \leq N} \mathcal{E}^{\mathrm{red}}\left(\gamma_{m}^{k}\right), \quad i_{m}^{\max }=\underset{1 \leq k \leq N}{\operatorname{argmax}} \mathcal{E}^{\mathrm{red}}\left(\gamma_{m}^{k}\right)
$$

4. if $\left\|E_{m}^{\max }-E_{m-1}^{\max }\right\|<\eta, M$ times consecutively, set $(\tilde{c}, \tilde{\varphi})=\gamma_{m}^{i_{m}^{\max }}$ and go to Step D (i.e. switch to a Newton-like algorithm);

5. for all $1 \leq k \leq N$, we search for an optimal step $0 \leq \alpha_{m}^{k} \leq 1$ and set

$$
d_{m}^{k}=-\alpha_{m}^{k} g_{m}^{k}, \quad D_{m}^{k}=-\alpha_{m}^{k} G_{m}^{k}
$$


6. we displace the nodes $\gamma_{m}^{k}$ along the descent directions:

$$
\begin{gathered}
c^{k, m+1}=\frac{1}{\left\|c^{k, m}+d_{m}^{k}\right\|}\left(c^{k, m}+d_{m}^{k}\right) \\
\varphi^{k, m+1}=\widetilde{\varphi}\left[\widetilde{\varphi}^{T} S \widetilde{\varphi}\right]^{-1 / 2} \quad \text { with } \quad \widetilde{\varphi}=\varphi^{k, m+1}+D_{m}^{k} ;
\end{gathered}
$$

7. we reparametrize the path. For this purpose, define the length of the discrete path by

$$
L=\sum_{k=1}^{N+1}\left\|c^{k, m+1}-c^{k-1, m+1}\right\|+\left\|\varphi^{k, m+1}-\varphi^{k-1, m+1}\right\|
$$

and search for a sequence of points $\gamma_{m+1}\left(t_{k}\right)$ of the continuous path $\gamma^{k+1}(t)$ satisfying

$$
\left\|\gamma_{m+1}\left(t_{k+1}\right)-\gamma_{m+1}\left(t_{k}\right)\right\| \in\left[L / N, L / N+\varepsilon^{\prime}\right]
$$

where $\varepsilon^{\prime}$ is a small enough threshold. The points $\gamma_{m+1}\left(t_{k}\right)$ define a reparametrized path $\gamma_{m+1}^{\text {rep }}(t)$; we finally set

$$
\gamma_{m+1}^{k}=\gamma_{m+1}^{\mathrm{rep}}(k / N)
$$

8. we set $m=m+1$ and return to step C.1.

Step D: we use the Newton algorithm described in Appendix, with $(\tilde{c}, \tilde{\varphi})$ as initial guess, to solve (24).

\subsection{Numerical results}

In this section, we present numerical results concerning the calculation of the first singlet excited state of some two-electrons systems, namely the $\mathrm{H}_{2}$ molecule and Helium-like atoms.

These results have been obtained with a Scilab [19] program, interfaced with a few $\mathrm{C}$ routines aiming in particular at speeding up the tensor-matrix products (21). Let us mention that the overlap matrices $S$, the core hamiltonians $h$, and the bielectronic integral tensors $W$ have been extracted from Gaussian 98 calculations [17].

\subsubsection{The $H_{2}$ molecule}

We have computed the first singlet excited state of the $H_{2}$ molecule, for various interatomic distances $R$. We have used for these calculations the double zeta Dunning's correlation consistent atomic basis set (CC-PVDZ), for which $N_{b}=10$. The number of iterations in Step C (path optimization) necessary to reach a given convergence criterion strongly depends on the choice of the initial guess. In that respect, the randomly perturbed initial paths constucted in Step B of our algorithm are of better quality than the one given by 
formula (26). Let us mention that one iteration of the path optimization procedure for $N_{b}=10$ and $N=100$ is about five seconds long on a Pentium IV $2.4 \mathrm{GHz}$.

Let us first analyze the results for a fixed interatomic distance equal to $R=0.5 \AA$. The energy profiles of the successive paths generated by the path optimization procedure (Step C) have been reported on the same graph (Figure 2). One can see that the energy profile of the initial trial path is a single hump and that the energy profiles of the iterates progressively turn into a double hump shape. The energy profiles of the earlier iterates have a rough shape for the initial trial path results from a stochastic local deformation of a reference path (Step $\mathrm{B}$ of the algorithm). The optimization process rapidly smoothes the trial path. Notice that due to the reparametrization procedure, the graph of $\mathcal{E}^{\text {red }}\left(\gamma_{k}\right)$ is not necessarily below the graph of $\mathcal{E}^{\text {red }}\left(\gamma_{k-1}\right)$.

The optimal path $\gamma$ obtained with our algorithm exhibits a double hump energy profile (see Figure 3) with two local maxima. Let us point out that we have run on this case many tests with different stochastic initial trial paths; we have always obtained a double hump profile at convergence.

Our method thus provides two saddle points of Morse index equal to one. According to our definition of MCSCF excited states, the one of higher energy (denoted by $M$ on Figure 3 ) corresponds to the first singlet excited state. On the other hand, the saddle point $M^{\prime}$ cannot be interpreted as a MCSCF excited state; it is rather a spurious solution of the MCSCF equations (24). Notice that the energy of $M^{\prime}$ corresponds to the first eigenvalue of the matrix $H(\varphi)$ appearing in equation (24), whereas the energy of $M$ corresponds to the second eigenvalue of $H(\varphi)$. We therefore obtain $\lambda_{2}^{K}=\mu_{2}^{K}$ in this case.

Let us now display the vectors $c$ for the three points $I, M$ and $M^{\prime}$ introduced in Figure 3 (of course $c_{F}=-c_{I}$ )

$$
c_{I}=\left[\begin{array}{c}
0.9953010 \\
-0.0369945 \\
-0.0087985 \\
-0.0369945 \\
-0.0441540 \\
-0.0441540 \\
-0.0498874 \\
-0.0088056 \\
-0.0088057 \\
-0.0042793
\end{array}\right] \quad c_{M}=\left[\begin{array}{c}
0.7009818 \\
0.7009818 \\
-0.0139024 \\
-0.0536869 \\
-0.0458169 \\
-0.0536868 \\
-0.0942362 \\
-0.0141266 \\
-0.0074823 \\
-0.0074823
\end{array}\right] \quad c_{M^{\prime}}=\left[\begin{array}{c}
-0.7069785 \\
0.7072304 \\
-1.02910^{-08} \\
-0.0021659 \\
-1.15910^{-08} \\
-1.32110^{-08} \\
0.0009807 \\
-8.25210^{-09} \\
0.0009807 \\
-8.25210^{-09}
\end{array}\right]
$$

It appears that $c_{M}$ possesses two dominant coefficients. This shows the usefulness of the MCSCF method for the calculation of excited states: the Hartree-Fock method is not able to correctly describe such a two-configuration state (recall that the square of the coefficients of $c$ are the weights of the different configurations of the multiconfiguration wavefunction). The structure of $c_{M^{\prime}}$ is very similar to the one of $c_{M}$, apart from a change of sign.

We can have an insight into the shape of the optimal path $\gamma$ by displaying the projection of $\gamma$ on several 3D representative subspaces selected by principal components analysis. Figure 4 shows the projection of the component $c(t)$ of $\gamma(t)$ as well as that of three different columns of $\varphi(t)$ (each of them representing a partially occupied one-electron wavefunction). The 


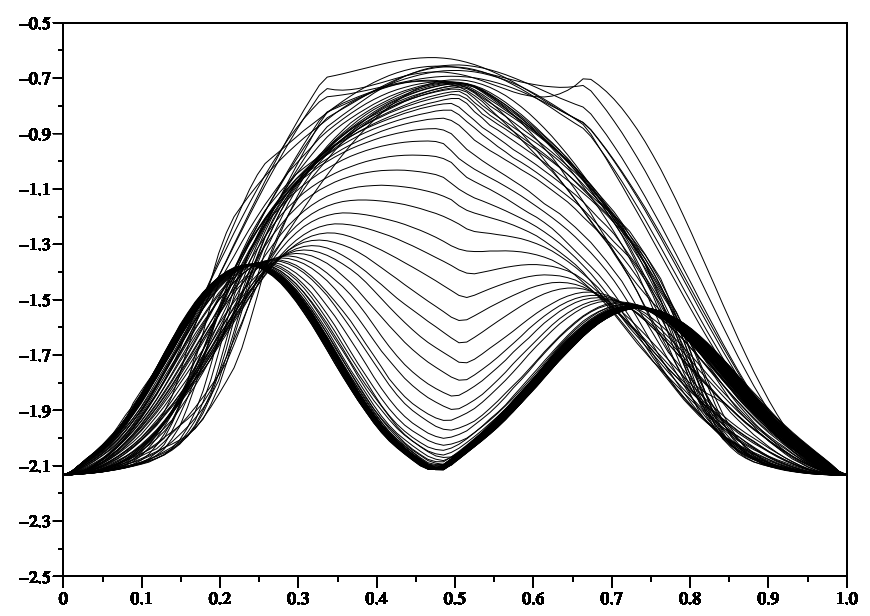

Figure 2: Energy profiles of the successive paths generated by the path optimization procedure $\left(H_{2}\right.$ molecule, interatomic distance equal to $0.5 \AA$ ).

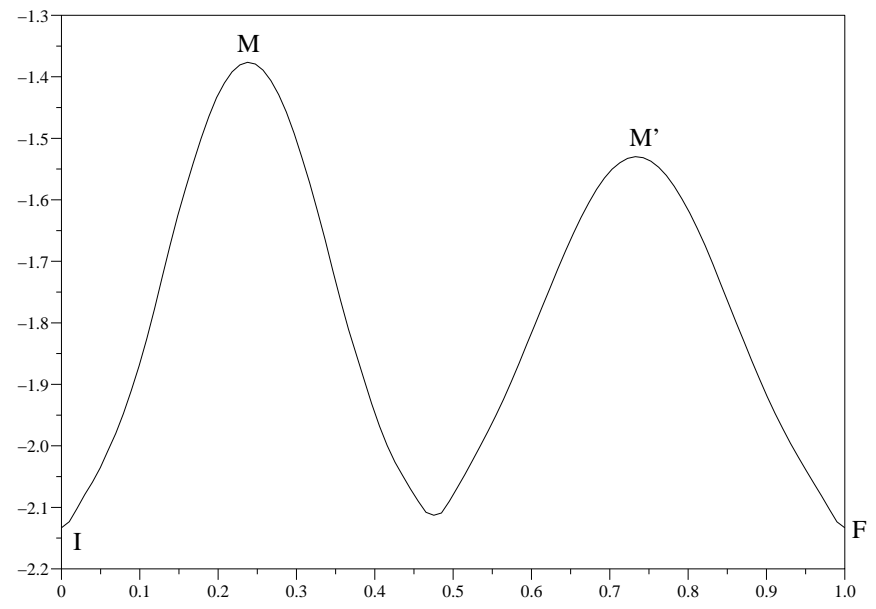

Figure 3: Path at convergence for $H_{2}$ with $R=0.5 \AA$.

$\mathrm{RR} \mathrm{n}^{\circ} 5289$ 
approximate location of the points $I, M, M^{\prime}$ and $F$ introduced in Figure 3 are reported on the graphs.

Let us now present our results for different values of the interatomic distance $R$. Figure 5 puts together three potential energy surfaces (PES) of $H_{2}$ : the Hartree-Fock and MCSCF ground state PES and the first MCSCF singlet excited state PES.

For values of $R$ in the range [1.5 $\AA$; $2.5 \AA$ ] , the optimal path exhibits the same characteristics as for the case $R=0.5 \AA$ reported above. However, the optimal path is more difficult to obtain than for smaller values of $R$. We have actually observed that in this range of values of $R$, the choice of the convergence criteria plays a crucial role in the quality of the results. Indeed, the difference $\| \max \left(\mathcal{E}^{\text {red }}\left(\gamma_{k}\right)\right)-\max \left(\mathcal{E}^{\text {red }}\left(\gamma_{k+1}\right) \|\right.$ can be very small during many consecutive iterations, just as if convergence was reached. But if we run many additional iterations, the algorithm finally escapes this trap and converges toward the (supposed) optimal path. We have observed that such a sequence of small changes in $\max \left(\mathcal{E}^{\text {red }}\left(\gamma_{k}\right)\right)$ occurs when the energy profile of the trial path turns from a single hump shape into a double hump shape (see Figure 6).

Many solutions can be proposed to avoid this drawback. One of them consists in using a continuation method. Actually, there are two ways for "propagating" an optimal path by a continuation method. The first way is to choose for the continuation parameter, the interatomic distance $R$ (i.e. inject the optimal path obtained for $R=R_{0}$ as initial guess in the calculation for $R=R_{0}+d R$ ). The second way is to introduce an additional parameter $s$, varying in the range $[0 ; 1]$, allowing to progressively switch on the electronic interaction term. For instance, one can simply multiply by $s$ the second term of the right-hand side of (20). When $s=0$, the two electrons are independent and an optimal path is easy to construct. We have observed that an efficient numerical strategy consists in making the continuation parameter $s$ increase logarithmically from 0 to 0.1 , then linearly from 0.1 to 1. Numerical simulations also show that it is more efficient to progressively turn on the electronic interaction than to use the interatomic distance as a continuation parameter. The former method has thus been used in the calculation of the excited state PES of the $\mathrm{H}_{2}$ molecule for values of $R$ in the range $[1.5 ; 2.5]$.

For $R=1.5$ and $R=2$, the first singlet excited state that we obtain with our method corresponds to the first eigenvalue of the matrix $H_{\Phi}$ (see formula (24). This means that, using the notations of Sections 2.1 and 2.2, we have in these cases

$$
\lambda_{2}^{K}<\mu_{2}^{K} .
$$

Therefore, the definition (15) of MCSCF excited states provides better results than the usual definition (11).

We notice that when $R$ grows, the values of the local maxima $M$ and $M^{\prime}$ get closer and closer, until some critical value of $R$ (between 2 and 2.5) for which both values are equal. Beyond this critical value, we obtain, depending on the initial guess, optimal paths that have either one or two humps and give the same excited state (see Figure 7). 

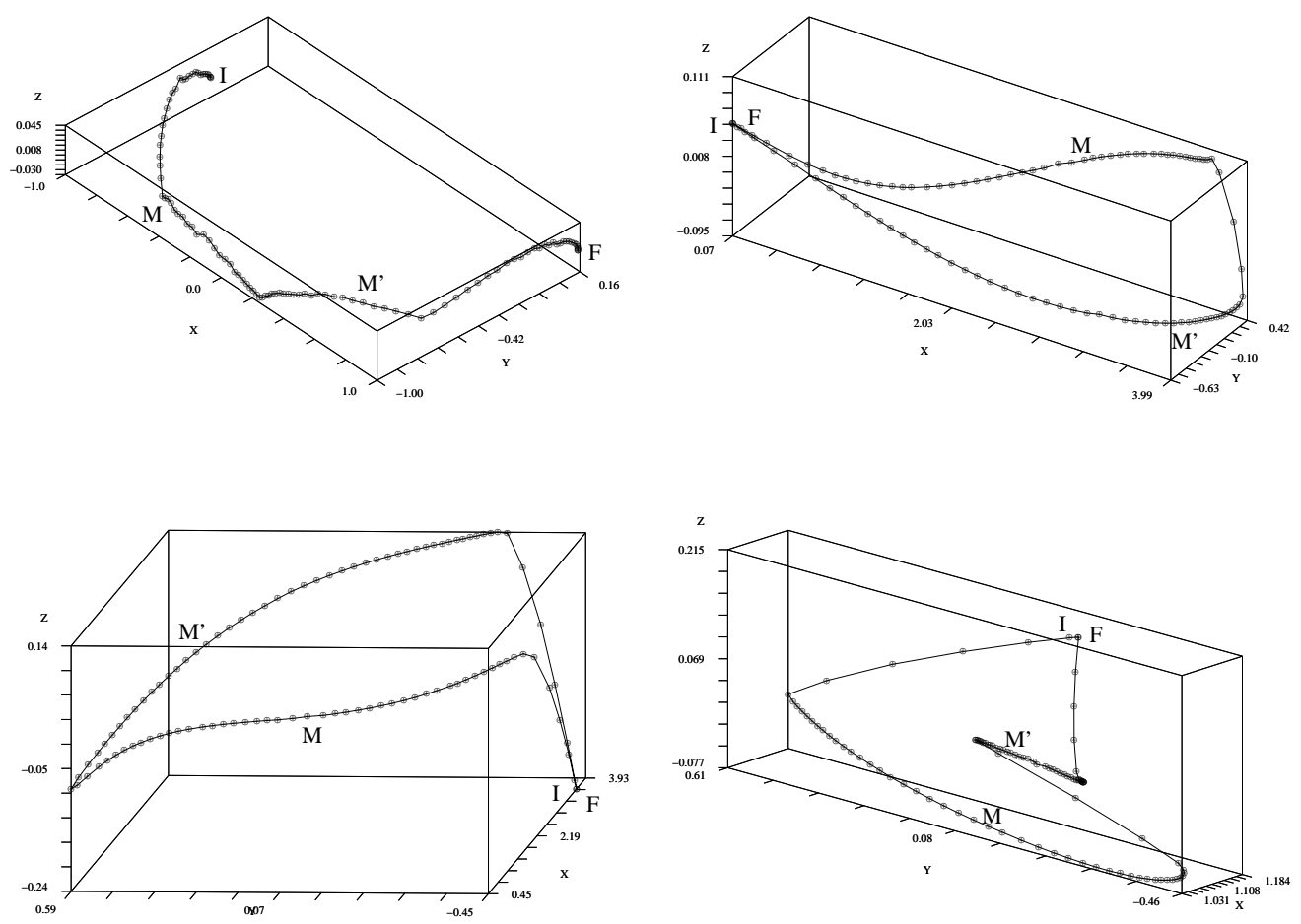

Figure 4: Projections of $c(t)$ (top left), $\varphi_{1}$ (top right), $\varphi_{2}$ (bottom left) and $\varphi_{7}$ (bottom right) on $3 \mathrm{D}$ representative subspaces selected by principal components analysis.

$\mathrm{RR} \mathrm{n}^{\circ} 5289$ 


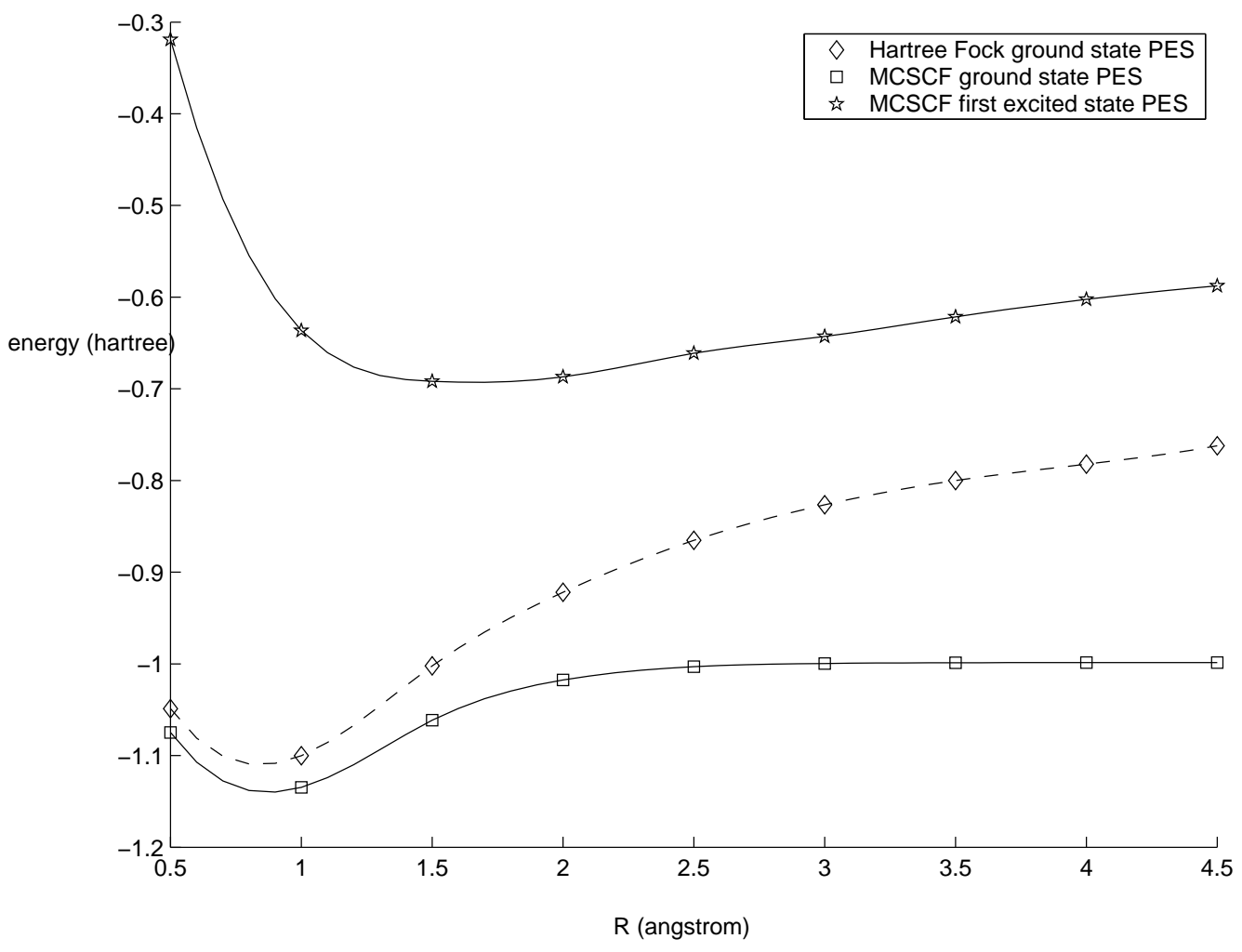

Figure 5: Potential energy surfaces (PES) of the $H_{2}$ molecule. 
Our numerical experiments thus show that there is a bifurcation phenomenon between single and double hump paths, in the vicinity of the minimum of the first excited state PES. It apparently corresponds to situations for which our method gives better results than the other methods currently used in Computational Chemistry.

\subsubsection{Helium-like atoms}

We have also tested our algorithm on the calculation of the first singlet excited state of Helium-like atoms (one nucleus of charge $Z \geq 2$ and two electrons). For these calculations, we have used a simple basis set made of the first six radial eigenstates of the corresponding Hydrogen-like atom (one nucleus of the same charge $Z$ and one electron), that are known analytically (see e.g. [28]). The results are shown on Table 1 for the values $Z=2,3,4$. It is to be noticed that the energy profiles of the optimal paths present double humps. This is consistent with the results obtained with the $\mathrm{H}_{2}$ molecule for small interatomic distances. The energy profiles of the sequence of paths obtained during the optimization process are put together on Figure 8.

\section{Appendix: the Newton algorithm}

We describe here the Newton-like algorithm which is used in our method. Define

$$
P_{c}=1-c c^{T}
$$

the orthogonal projector on $\{c\}^{\perp}$,

$$
Q_{\varphi}=P_{\varphi}^{S}
$$

where $P_{\varphi}^{S}$ is defined in formula (27), and

$$
Q_{\varphi}^{*} Z=Z-\frac{1}{2} S \varphi\left(\varphi^{T} Z+Z^{T} \varphi\right)
$$

the adjoint of $Q_{\varphi}$ for the euclidian scalar product. Note that $Q_{\varphi}$ is an orthogonal projector, only for the scalar product defined by the matrix $S$ (see page 20).

The Newton equations take the form, $(c, \varphi, \mu, \Lambda) \in \mathbb{R}^{K} \times M\left(N_{b}, N\right) \times \mathbb{R} \times M_{s}(K)$ being given,

$$
\left\{\begin{array}{l}
\text { Find }(d, \zeta, \nu, M) \in \mathbb{R}^{K} \times M\left(N_{b}, K\right) \times \mathbb{R} \times M_{s}(K) \text { such that } \\
{[H(\varphi)-\mu] d+\left[H^{\prime}(\varphi) \cdot \zeta\right] c=\nu c-(H(\varphi) \cdot c-\mu c)} \\
\mathcal{L}_{(c, \varphi, \mu, \Lambda)} \cdot(d, \zeta)=S \varphi M-\left(h \varphi C^{s}(c)^{2}+\mathcal{W}\left(\varphi C^{s}(c) \varphi^{T}\right) \varphi C^{s}(c)-S \varphi \Lambda\right) \\
c^{T} d=-\left(c^{T} c-1\right) \\
\varphi^{T} S \zeta+\zeta^{T} S \varphi=-\left(\varphi^{T} S \varphi-I_{K}\right)
\end{array}\right.
$$

RR $n^{\circ} 5289$ 


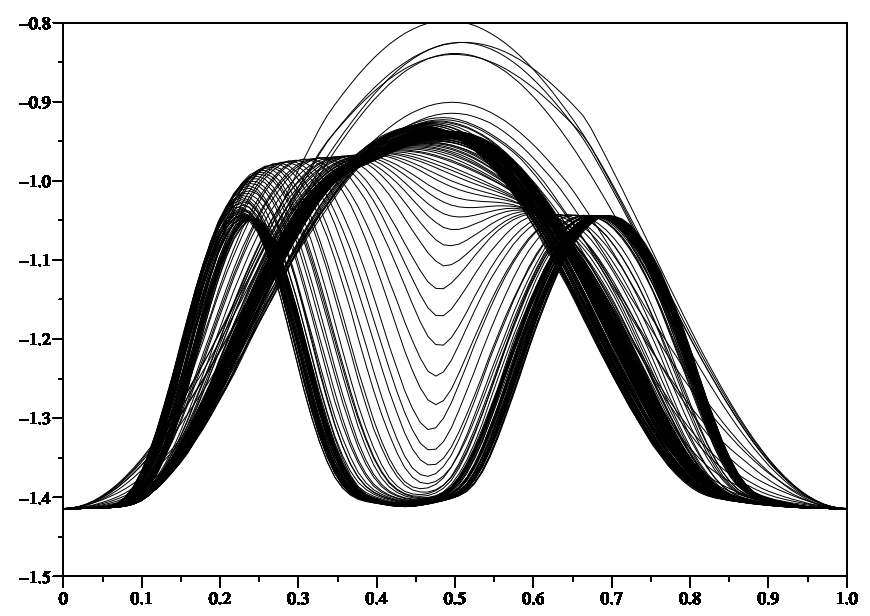

Figure 6: Iterates of $\gamma_{k}$ during the optimization process until convergence, for $R=1.5 \AA$.
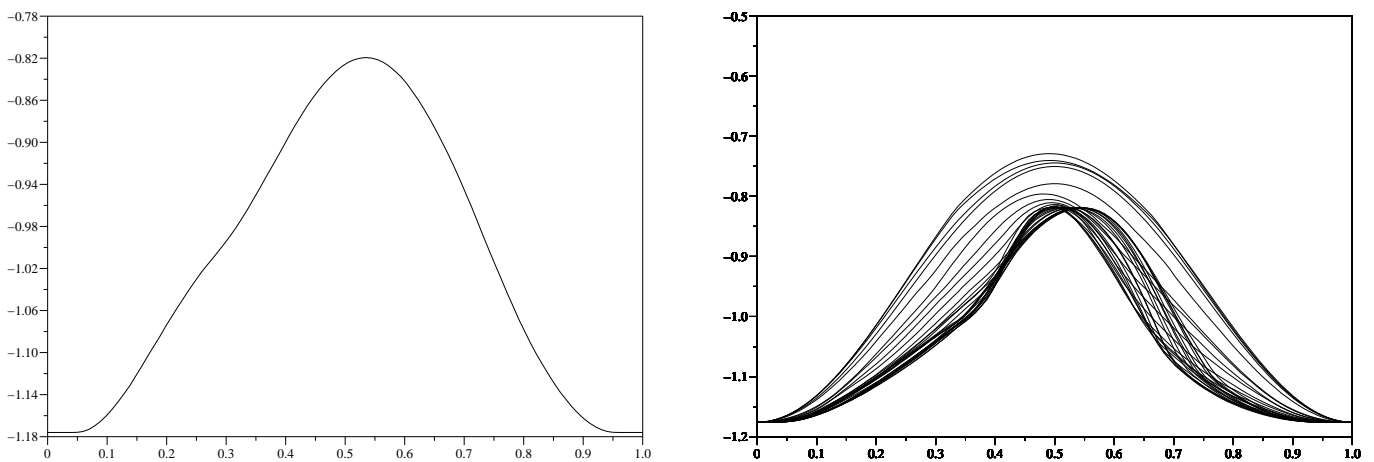

Figure 7: Path at convergence and iterates of the path during the optimization process for $H_{2}$ with $R=3 \stackrel{\circ}{\AA}$. 

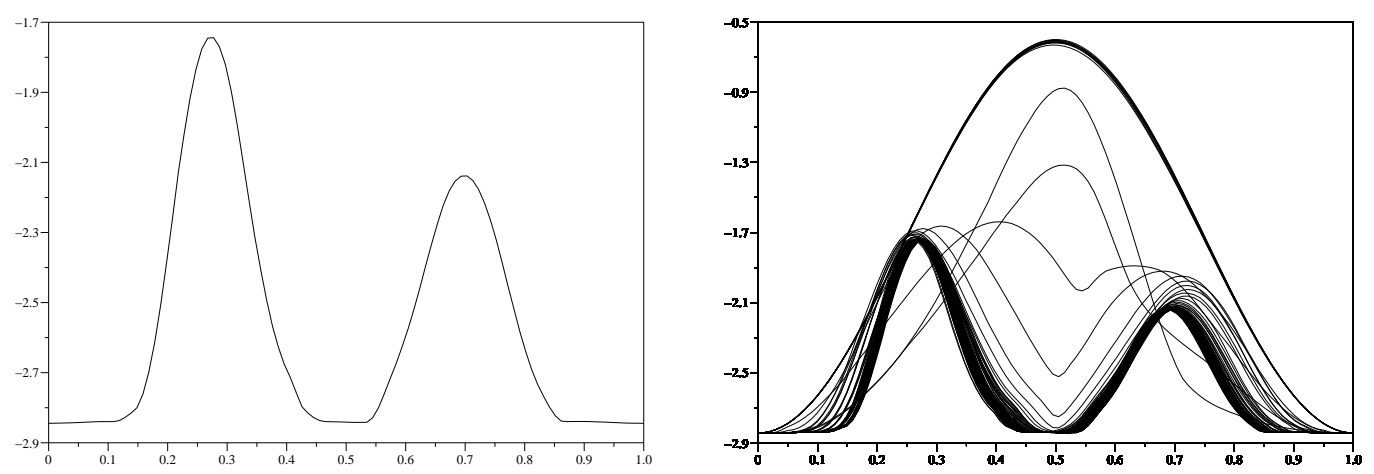

Figure 8: Optimal path and iterates of the path during the optimization process for the Helium atom.

\begin{tabular}{|l|c|c|c|}
\hline & $Z=2$ & $Z=3$ & $Z=4$ \\
\hline HF ground state energy & -2.835374 & -7.200180 & -13.570185 \\
MCSCF ground state energy & -2.844340 & -7.204910 & -13.573562 \\
MCSCF first singlet excited state & -1.741472 & -4.4432095 & -8.4031421 \\
\hline
\end{tabular}

Table 1: Results for Helium-like atoms (one nucleus of charge $Z$ and two electrons).

$\mathrm{RR} \mathrm{n}^{\circ} 5289$ 
with

$$
\begin{aligned}
\mathcal{L}_{(c, \varphi, \mu, \Lambda)} \cdot(d, \zeta)=h \zeta C^{s}(c)^{2} & +2 h \varphi C^{s}(c) C^{s}(d)+ \\
+\mathcal{W}\left(\varphi C^{s}(c) \zeta^{T}\right. & \left.+\zeta C^{s}(c) \varphi^{T}\right) \varphi C^{s}(c)+\mathcal{W}\left(\varphi C^{s}(c) \varphi^{T}\right) \zeta C^{s}(c)+ \\
& +\mathcal{W}\left(\varphi C^{s}(d) \varphi^{T}\right) \varphi C^{s}(c)+\mathcal{W}\left(\varphi C^{s}(c) \varphi^{T}\right) \varphi C^{s}(d)-S \zeta \Lambda .
\end{aligned}
$$

Note that

$$
\begin{aligned}
{\left[\left[H^{\prime}(\varphi) \cdot \zeta\right] c\right]_{i}=} & \sum_{j=1}^{K}\left[H^{\prime}(\varphi) \cdot \zeta\right]_{i j} c_{j} \\
= & 2\left(\zeta_{i}^{T} h \varphi_{i}+\varphi_{i}^{T} h \zeta_{i}\right) c_{i}+\left(\zeta_{i}^{T} \mathcal{W}\left(\varphi C^{s}(c) \varphi^{T}\right) \varphi_{i}\right. \\
& \left.+\varphi_{i}^{T} \mathcal{W}\left(\varphi C^{s}(c) \varphi^{T}\right) \zeta_{i}\right)+\sum_{j=1}^{K}\left(\zeta_{j}^{T} \mathcal{W}\left(\varphi_{i} \varphi_{i}^{T}\right) \varphi_{j}+\varphi_{j}^{T} \mathcal{W}\left(\varphi_{i} \varphi_{i}^{T}\right) \zeta_{j}\right) c_{j} \\
= & 2\left(\zeta_{i}^{T} h \varphi_{i}+\varphi_{i}^{T} h \zeta_{i}\right) c_{i}+\left(\zeta_{i}^{T} \mathcal{W}\left(\varphi C^{s}(c) \varphi^{T}\right) \varphi_{i}+\right. \\
& \left.\varphi_{i}^{T} \mathcal{W}\left(\varphi C^{s}(c) \varphi^{T}\right) \zeta_{i}\right)+\operatorname{tr}\left(\mathcal{W}\left(\varphi_{i} \varphi_{i}^{T}\right)\left(\varphi C^{s}(c) \zeta^{T}+\zeta C^{s}(c) \varphi^{T}\right)\right)
\end{aligned}
$$

Let us consider a MCSCF state $(c, \varphi) \in S^{K-1} \times \mathcal{W}_{K}^{N_{b}}$ satisfying the constraints, and let

$$
\mu=c^{T} H(\varphi) c, \quad \Lambda=\varphi^{T}\left(h \varphi C^{s}(c)^{2}+\mathcal{W}\left(\varphi C^{s}(c) \varphi^{T}\right) \varphi C^{s}(c)\right) .
$$

We define the linear map $\delta(\zeta): M\left(N_{b}, K\right) \rightarrow \mathbb{R}^{K}$ by

$$
\delta(\zeta)=-\left[P_{c}(H(\varphi)-\mu) P_{c}\right]^{-1} \cdot\left(P_{c}\left(\left[H^{\prime}(\varphi) \cdot \zeta\right] c\right)\right) .
$$

Notice that $\delta(\zeta)$ is well defined as soon as $H(\varphi)-\mu$ is invertible on $\{c\}^{\perp}$. Let be

$$
\alpha=\left[P_{c}(H(\varphi)-\mu) P_{c}\right]^{-1} \cdot\left(P_{c}([H(\varphi)-\mu] c)\right) .
$$

We then introduce

$$
L_{(c, \varphi, \mu, \Lambda)} \cdot \zeta=Q_{\varphi}^{*} \mathcal{L}_{(c, \varphi, \mu, \Lambda)}\left(\delta\left(Q_{\varphi} \zeta\right), Q_{\varphi} \zeta\right)
$$

and

$$
\begin{aligned}
b_{(c, \varphi, \mu, \Lambda)}=- & Q_{\varphi}^{*}\left(h \varphi C^{s}(c)^{2}+\mathcal{W}\left(\varphi C^{s}(c) \varphi^{T}\right) \varphi C^{s}(c)-S \varphi \Lambda\right. \\
& \left.-2 h \varphi C^{s}(c) C^{s}(\alpha)-\mathcal{W}\left(\varphi C^{s}(\alpha) \varphi^{T}\right) \varphi C^{s}(c)-\mathcal{W}\left(\varphi C^{s}(c) \varphi^{T}\right) \varphi C^{s}(\alpha)\right)
\end{aligned}
$$

INRIA 


\section{Newton algorithm}

Initial guess. Some $\left(c_{0}, \varphi_{0}\right) \in S^{K-1} \times \mathcal{W}_{K}^{N_{b}}$. Set $k=0$.

\section{Iterates.}

1. Set

$$
\mu_{k}=c_{k}^{T} H\left(\varphi_{k}\right) c_{k}, \quad \Lambda_{k}^{\prime}=\varphi_{k}^{T}\left\{h \varphi_{k} C^{s}\left(c_{k}\right)^{2}+\mathcal{W}\left(\varphi_{k} C^{s}\left(c_{k}\right) \varphi_{k}^{T}\right) \varphi_{k} C^{s}\left(c_{k}\right)\right\}
$$

and (the symmetry of $\Lambda_{k}$ is imposed)

$$
\Lambda_{k}=\frac{1}{2}\left(\Lambda_{k}^{\prime}+\left(\Lambda_{k}^{\prime}\right)^{T}\right) .
$$

2. Compute

$$
\begin{gathered}
r_{k}=H\left(\varphi_{k}\right) \cdot c_{k}-\mu_{k} c_{k} \\
R_{k}=h \varphi_{k} C^{s}\left(c_{k}\right)^{2}+\mathcal{W}\left(\varphi_{k} C^{s}\left(c_{k}\right) \varphi_{k}^{T}\right) \varphi_{k} C^{s}\left(c_{k}\right)-S \varphi_{k} \Lambda_{k} .
\end{gathered}
$$

If $\left\|r_{k}\right\| \leq \varepsilon_{c}$ and if $\left\|R_{k}\right\| \leq \varepsilon_{\varphi}$, STOP.

3. Use an orthonormalization method (Gramm-Schmidt for instance) to build a matrix $B_{k} \in M(K, K-1)$ such that $\left[c_{k} \mid B_{k}\right] \in U(K)$. Then, let

$$
\begin{gathered}
T_{k}=\left(B_{k}^{T}\left[H\left(\varphi_{k}\right)-\mu_{k}\right] B_{k}\right)^{-1}, \\
\alpha_{k}=B_{k} T_{k} B_{k}^{T} r_{k}
\end{gathered}
$$

and

$$
\begin{aligned}
b_{k}=-Q_{k}^{*}\left(R_{k}-2 h \varphi_{k} C^{s}\left(c_{k}\right) C^{s}\left(\alpha_{k}\right)\right) & \\
& \left.-\mathcal{W}\left(\varphi_{k} C^{s}\left(\alpha_{k}\right) \varphi_{k}^{T}\right) \varphi_{k} C^{s}\left(c_{k}\right)-\mathcal{W}\left(\varphi_{k} C^{s}\left(c_{k}\right) \varphi_{k}^{T}\right) \varphi_{k} C^{s}\left(\alpha_{k}\right)\right),
\end{aligned}
$$

with $Q_{k}^{*} Z=Z-\frac{1}{2} S \varphi_{k}\left(\varphi_{k}^{T} Z+Z^{T} \varphi_{k}\right)$

4. Solve the linear symmetric system

$$
L_{k} \zeta_{k}=b_{k},
$$

where $L_{k}=L_{\left(c_{k}, \varphi_{k}, \mu_{k}, \Lambda_{k}\right)}$. To this end, one can use for instance the GMRes method. To apply the operator $L_{k}$ to some vector $\zeta$, one follows : 
(a) Compute $Q_{k} \zeta$ where $Q_{k}=Q_{\varphi_{k}}$.

(b) Compute

$$
\delta\left(Q_{k} \zeta\right)=-B_{k} T_{k} B_{k}^{T}\left(\left[H^{\prime}\left(\varphi_{k}\right) \cdot\left(Q_{k} \zeta\right)\right] c_{k}\right)
$$

(c) Compute $L_{k} \zeta=Q_{k}^{*} \mathcal{L}_{\left(c_{k}, \varphi_{k}, \mu_{k}, \Lambda_{k}\right)}\left(\delta\left(Q_{k} \zeta\right), Q_{k} \zeta\right)$ using formula (29).

5. Set

$$
\begin{gathered}
\zeta_{k}=Q_{k} \zeta_{k}, \\
d_{k}=-B_{k} T_{k} B_{k}^{T}\left(\left[H^{\prime}\left(\varphi_{k}\right) \cdot \zeta_{k}\right] c_{k}\right)-\alpha_{k} .
\end{gathered}
$$

6. Set

$$
\begin{aligned}
c_{k+1}= & \left\|c_{k}+d_{k}\right\|^{-1}\left(c_{k}+d_{k}\right), \\
& \widetilde{\varphi}_{k+1}=\varphi_{k}+\zeta_{k}, \\
\varphi_{k+1}= & \widetilde{\varphi}_{k+1}\left(\widetilde{\varphi}_{k+1}^{T} S \widetilde{\varphi}_{k+1}\right)^{-1 / 2} .
\end{aligned}
$$

7. Set $k=k+1$ and return to Step 1 .

This Newton-like method has been used on the one hand to find the MCSCF ground state, and on the other hand to achieve the convergence of the mountain pass point.

For the minimization of $\mathcal{E}^{\text {red }}$, the step 6 has to be improved by using a line search method to minimize the energy on the curve $(c(t), \varphi(t))_{t \in[0,1]}$ with

$$
\begin{gathered}
c(t)=\left\|c_{k}+t d_{k}\right\|^{-1}\left(c_{k}+t d_{k}\right), \\
\widetilde{\varphi}(t)=\varphi_{k}+t \zeta_{k}, \\
\varphi(t)=\widetilde{\varphi}(t)\left(\widetilde{\varphi}(t)^{T} S \widetilde{\varphi}(t)\right)^{-1 / 2} .
\end{gathered}
$$

Acknowledgment: We would like to thank Claude Le Bris and Éric Séré for useful discussions and advice. 


\section{References}

[1] T. Ando, Properties of fermions density matrices, Rev. Mod. Phys. 35 (1963), no. 3, 690-702.

[2] P.W. Atkins and R.S. Friedman, Molecular quantum mechanics, third ed., Oxford University Press, 1997.

[3] M. Born and R. Oppenheimer, Quantum theory of molecules, Ann. Physics 84 (1927), 457-484.

[4] E. Cancès, M. Defranceschi, W. Kutzelnigg, C. Le Bris, and Y. Maday, Computational quantum chemistry: a primer, Handbook of Numerical Analysis, vol. X, pp. 3-270, Elsevier, 2003.

[5] L.M. Cheung, S.T. Elbert, and K. Ruedenberg, MCSCF optimization through combined use of natural orbital and the Brillouin-Levy-Berthier theorem, Int. J. Quantum Chem. 16 (1979), 1069-1101.

[6] S.S-L. Chiu, J.W. McDouall, and I.H. Hiller, Prediction of whole reaction paths for large molecular systems, J. Chem. Soc. Faraday Trans. 90 (1994), no. 12, 1575-1579.

[7] Y.S. Choi and P.J. McKenna, A mountain pass method for the numerical solution of semilinear elliptic problems, Nonlinear Anal. 20 (1993), no. 4, 417-437.

[8] Y.S. Choi, P.J. McKenna, and M. Romano, A mountain pass method for the numerical solution of semilinear wave equations, Numer. Math. 64 (1993), no. 4, 487-509.

[9] A.J. Coleman, Structure of fermions density matrices, Rev. Mod. Phys. 35 (1963), no. $3,668-689$.

[10] A.J. Coleman and V.I. Yukalov, Reduced density matrices: Coulson's challenge, Springer Verlag, 2000.

[11] R. Czerminski and R. Elber, Self-avoiding walk between two fixed points as a tool to calculate reaction paths in large molecular systems, Int. J. Quantum Chemistry 24 (1990), 167-185.

[12] R.M. Dreizler and E.K.U. Gross, Density functional theory, Springer Verlag, 1990.

[13] R.H.A. Eade and M.A. Robb, Direct minimization in MCSCF theory. The quasi-Newton method., Chem. Phys. Letters 83 (1981), no. 2, 362-368.

[14] A. Edelman, T.A. Arias, and S.T. Smith, The geometry of algorithms with orthogonality constraints, SIAM J. Matrix Anal. Appl. 20 (1998), no. 2, 303-353.

[15] R. Elber and M. Karplus, A method for determining reaction paths in large molecules: application to myoglobin, Chem. Phys. Letters 139 (1987), no. 5, 375-380.

RR $n^{\circ} 5289$ 
[16] G. Friesecke, The multiconfiguration equations for atoms and molecules: charge quantization and existence of solutions, Arch. Rat. Mech. Analysis 169 (2003), 35-71.

[17] M.J. Frisch, G.W. Trucks, H.B. Schlegel, G.E. Scuseria, M.A. Robb, J.R. Cheeseman, V.G. Zakrzewski, J.A. Montgomery, R.E. Stratmann, J.C. Burant, S. Dapprich, J.M. Millam, A.D. Daniels, K.N. Kudin, M.C. Strain, O. Farkas, J. Tomasi, V. Barone, M. Cossi, R. Cammi, B. Mennucci, C. Pomelli, C. Adamo, S. Clifford, J. Ochterski, G.A. Petersson, P.Y. Ayala, Q. Cui, K. Morokuma, D.K. Malick, A.D. Rabuck, K. Raghavachari, J.B. Foresman, J. Cioslowski, J.V. Ortiz, B.B. Stefanov, G. liu, A. Liashenko, P. Piskorz, I. Kpmaromi, G. Gomperts, R.L. Martin, D.J. Fox, T. Keith, M.A. Al-Laham, C.Y. Peng, A. Nanayakkara, C. Gonzalez, M. Challacombe, P.M.W. Gill, B.G. Johnson, W. Chen, M.W. Wong, J.L. Andres, M. Head-Gordon, E.S. Replogle, and J.A. Pople, Gaussian 98 (Revision A.7), Gaussian Inc., Pittsburgh PA, 1998.

[18] N. Ghoussoub, Duality and perturbation methods in critical point theory, Cambridge University Press, 1993.

[19] C. Gomez, C. Bunks, J.P. Chancelier, F. Delebecque, M. Goursat, R. Nikoukhah, and S. Steer, Engineering and scientific computing with scilab, Birkauser, 1999.

[20] M. Head-Gordon, Quantum chemistry and molecular processes, J. Phys. Chem. 100 (1996), 13213-13225.

[21] W.J. Hehre, L. Radom, P.v.R. Schleyer, and J.A. Pople, Ab initio molecular orbital theory, Wiley, 1986.

[22] G. Henkelman, G. Jóhannesson, and H. Jónsson, Methods for finding saddle points and minimum energy paths, Ed. S. D. Schwartz (Kluwer Academic Publishers), 2000.

[23] G. Henkelman and H. Jónsson, A dimer method for finding saddle points on high dimensional potential surfaces using only first derivatives, J. Chem. Physics 111 (1999), no. $15,7010-7022$.

[24] _ Improved tangent estimate in the nudged elastic band method for finding minimum energy paths and saddle points, J. Chem. Physics 113 (2000), no. 22, 9978-9985.

[25] G. Henkelman, H. Jónsson, and B.P. Uberuaga, A climbing image nudged elastic band method for finding saddle points and minimum energy paths, J. Chem. Physics 113 (2000), no. 22, 9901-9904.

[26] P.G. Jasien and R. Shepard, A general polyatomic potential energy surface fitting method, Int. J. Quantum Chemistry 22 (1988), 183-198.

[27] W. Kohn and L.J. Sham, Self-consistent equations including exchange and correlation effects, Phys. Rev. 140 (1965), A1133-A1138. 
[28] L.D. Landau and E.H. Lifchitz, Quantum mechanics, Pergamon Press, 1977.

[29] C. Le Bris, A general approach for multiconfiguration methods in quantum molecular chemistry, Ann. Inst. H. Poincaré Anal. Non linéaire 11 (1994), no. 6, 441-484.

[30] J.F. Léon, Excited states for Coulomb systems in the Hartree-Fock approximation, Comm. Math. Phys. 120 (1988), no. 2, 261-268.

[31] M. Lewin, Solutions of the multiconfiguration equations in quantum chemistry, Arch. Rat. Mech. Anal. 171 (2004), no. 1, 83-114.

[32] A.S. Lewis and M.L. Overton, Eigenvalue optimization, Acta Num. 5 (1996), 149-190.

[33] E.H. Lieb and B. Simon, The Hartree-Fock theory for Coulomb systems, Commun. Math. Phys. 53 (1977), 185-194.

[34] P.L. Lions, Solutions of Hartree-Fock equations for Coulomb systems, Commun. Math. Phys. 109 (1987), 33-87.

[35] P.O. Löwdin, Quantum theory of many-particle systems. I. Physical interpretations by mean of density matrices, natural spin-orbitals, and convergence problems in the method of Configurational Interaction, Phys. Rev. 97 (1955), no. 6, 1474-1489.

[36] — Quantum theory of many-particle systems. II. Study of the ordinary HartreeFock approximation, Phys. Rev. 97 (1955), no. 6, 1490-1508.

[37] _ Quantum theory of many-particle systems. III. Extension of the Hartree-Fock scheme to include degenerate systems and correlation effects, Phys. Rev. 97 (1955), no. $6,1509-1520$.

[38] _ Correlation problem in many-electron quantum mechanics. I. Review of different approaches and discussion of some current ideas, Adv. Chem. Phys. 2 (1959), $207-322$.

[39] M.A. Robb M. Frisch, I.N. Ragazos and H.B. Schlegel, An evaluation of three direct mcscf procedures, Chem. Phys. Letters 189 (1992), no. 6, 524-528.

[40] W. Quapp and D. Heidrich, Analysis of the concept of minimum energy path on the potential energy surface of chemically reactiong systems, Theoret. Chim. Acta 66 (1984), $245-260$.

[41] W. Quapp, H. Hirsch, O. Imig, and D. Heidrich, Searching for saddle points of potential energy surfaces by following a reduced gradient, J. Comput. Chem. 19 (1998), no. 9, $1087-1100$.

[42] M. Reed and B. Simon, Methods of modern mathematical physics, vol. IV - Analysis of Operators, Academic Press, 1978.

RR $n^{\circ} 5289$ 
[43] B. O. Roos, The Complete Active Space Self-Consistent Field method and its applications in electronic structure calculation, Ab Initio Methods in Quantum Chemistry - II in Adv. Chem. Phys. 69 (1987), 399-446.

[44] H.B. Schlegel, Optimization of equilibrium geometries and transition structures, Adv. Chem. Phys. 67 (1987), 249-286.

[45] R. Shepard, The Multiconfiguration Self-Consistent Field method, Ab Initio Methods in Quantum Chemistry - II in Adv. Chem. Phys. 69 (1987), 63-200.

[46] E. Weinan, R. Weiqing, and E. Vanden-Eijnden, String method for the study of rare events, Phys. Rev. B 66 (2002), 052301.

[47] H.-J. Werner, Matrix-formulated direct Multiconfiguration Self-Consistent Field and Multiconfiguration Reference Configuration-Interaction methods, Ab Initio Methods in Quantum Chemistry - II in Adv. Chem. Phys. 69 (1987), 1-62.

[48] H.-J. Werner and W. Meyer, A quadratically convergent Multiconfiguration-SelfConsistent Field method with simultaneous optimization of orbitals and CI coefficients, J. Chem. Phys. 73 (1980), no. 5, 2342-2356.

[49] G. M. Zhislin, Discussion of the spectrum of Schrödinger operators for systems of many particles. (In Russian), Trudy Moskovskogo matematiceskogo obscestva 9 (1960), 81120. 


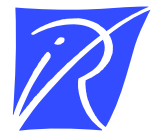

Unité de recherche INRIA Rocquencourt

Domaine de Voluceau - Rocquencourt - BP 105 - 78153 Le Chesnay Cedex (France)

Unité de recherche INRIA Futurs : Parc Club Orsay Université - ZAC des Vignes

4, rue Jacques Monod - 91893 ORSAY Cedex (France)

Unité de recherche INRIA Lorraine : LORIA, Technopôle de Nancy-Brabois - Campus scientifique

615, rue du Jardin Botanique - BP 101 - 54602 Villers-lès-Nancy Cedex (France)

Unité de recherche INRIA Rennes : IRISA, Campus universitaire de Beaulieu - 35042 Rennes Cedex (France)

Unité de recherche INRIA Rhône-Alpes : 655, avenue de l'Europe - 38334 Montbonnot Saint-Ismier (France)

Unité de recherche INRIA Sophia Antipolis : 2004, route des Lucioles - BP 93 - 06902 Sophia Antipolis Cedex (France)

INRIA - Domaine de Voluceau - Rocquencourt, BP 105 - 78153 Le Chesnay Cedex (France)

http://www.inria.fr

ISSN 0249-6399 